\title{
Implications of Bid Structures on the Offering Strategies of Merchant Energy Storage Systems
}

Gustavo De Vivero-Serrano, Kenneth Bruninx and Erik Delarue

TME WORKING PAPER - Energy and Environment Last update: April 2019

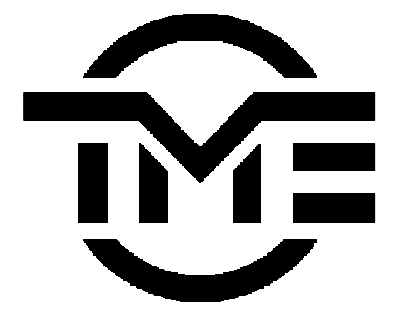

An electronic version of the paper may be downloaded from the TME website:

http://www.mech.kuleuven.be/tme/research/ 


\title{
Implications of Bid Structures on the Offering Strategies of Merchant Energy Storage Systems
}

\author{
Gustavo De Vivero-Serrano ${ }^{\mathrm{a}, \mathrm{b}}$, Kenneth Bruninx ${ }^{\mathrm{a}, \mathrm{b}, \mathrm{c}}$, Erik Delarue $^{\mathrm{a}, \mathrm{b}, *}$ \\ ${ }^{a}$ Division of Applied Mechanics $\&$ Energy Conversion, Mechanical Engineering, KU Leuven \\ ${ }^{b}$ EnergyVille, a joint venture of KU Leuven, VITO \& IMEC \\ ${ }^{c}$ VITO, The Flemish Institute for Technolgical Research \\ Leuven (KU Leuven), Genk (Energyville), Mol (VITO), Belgium
}

\begin{abstract}
Energy storage Systems (ESS) may play a pivotal role in the cost-efficient integration of renewable energy sources. Integrating large volumes of grid-scale energy storage into electricity markets, however, raises questions related to their profitability and impact on electricity prices. This paper explores the implications of different bid structures on the strategic behavior of a storage owner that participates in the wholesale market and is able to influence prices to maximize its profits. We conduct a comparative analysis on four bid structures present in existing markets and the scientific literature: (i) simple quantity-based bids, (ii) simple price-based bids, (iii) price-quantity pairs bids and (iv) complex bidding in which the ESS owner provides full information of its technical characteristics through the bids. The decision problem of the ESS owner is formulated as a bilevel programming model, where the upper-level problem represents the profit maximization of the ESS and the lower-level problem simulates possible market clearing outcomes. These bilevel models are reformulated as equivalent mixed-integer linear programming problems by means of the Karush-KuhnTucker optimality conditions, the strong duality theorem and the Big-M method. In a case study, we show that when the ESS owner is uncertain about the market clearing outcome, the bid structure affects the ESS's profitability and, to a lesser extent, the market's efficiency and the system's generation costs.
\end{abstract}

Keywords: Energy storage, bid structures, offering strategy, MPEC, electricity market design.

\footnotetext{
*Corresponding author

Email address: erik.delarue@kuleuven.be (Erik Delarue)
} 


\section{Nomenclature}

Below, the sets, parameters and variables used in this paper are listed. The list of dual variables is limited to those that appear in the main body of the manuscript. All other dual variables and their corresponding constraints are discussed in Appendix A.

\section{Sets}

$\mathcal{I} \quad$ Conventional generation units indexed by $i$.

$\mathcal{J} \quad$ Renewable units indexed by $j$.

$\mathcal{K} \quad$ Scenarios indexed by $k$.

$\mathcal{S} \quad$ Storage units indexed by $s$.

$\mathcal{T} \quad$ Time steps indexed by $t$.

\section{Parameters}

$\delta_{s}^{c}, \delta_{s}^{d} \quad$ Charging and discharging efficiency of storage unit $s(-)$.

$\bar{g}_{i} \quad$ Maximum generation capacity of conventional unit $i$ (MW).

$\bar{r}_{s}, \bar{q}_{s} \quad$ Maximum charging $(r)$ and discharging $(q)$ capacity of storage unit $s(\mathrm{MW})$.

$\bar{w}_{j, t, k} \quad$ Maximum generation output of renewable unit $j$ at time step $t$ in scenario $k(\mathrm{MW})$.

П $\quad$ Wholesale price cap $(€ / M W h)$.

$\underline{v}_{s}, \bar{v}_{s} \quad$ Minimum and maximum energy storage capacity of unit $s$ (MWh).

$c^{\phi} \quad$ Load shedding cost $(€ / M W h)$.

$c_{i, t} \quad$ Variable cost of conventional unit $i$ at time step $t(€ / \mathrm{MWh})$.

$D_{t} \quad$ Electricity demand at time step $t(\mathrm{MW})$.

$p_{k} \quad$ Probability of occurrence of scenario $k(-)$.

$v_{s}^{0} \quad$ Energy stored in storage unit $s$ at time step $t=0$ (MWh).

\section{Primal variables}

$\beta_{s, t}^{r}, \beta_{s, t}^{q} \quad$ Charging $\left({ }^{r}\right)$ and discharging $\left({ }^{q}\right)$ price bids by storage system $s$ at time step $t(€ / \mathrm{MWh})$.

$\phi_{t, k} \quad$ Load shedding at time step $t$ in scenario $k$ (MWh).

$g_{i, t, k} \quad$ Electricity generated by conventional unit $i$ at time step $t$ in scenario $k$ (MWh).

$q_{s, t, k} \quad$ Electricity generated by storage unit $s$ at time step $t$ in scenario $k$ (MWh). 


\begin{tabular}{|c|c|}
\hline$r_{s, t, k}$ & Electricity consumed by storage unit $s$ at time step $t$ in scenario $k$ (MWh). \\
\hline$v_{s, t, k}$ & Energy content in storage unit $s$ at time step $t$ in scenario $k$ (MWh). \\
\hline$w_{j, t, k}$ & Electricity generated by renewable unit $j$ at time step $t$ in scenario $k(\mathrm{MWh})$. \\
\hline$y_{s, t}, x_{s, t}$ & $\begin{array}{l}\text { Charging }(y) \text { and discharging }(x) \text { quantity bid by storage system } s \text { at time } \\
\text { step } t(\mathrm{MWh}) \text {. }\end{array}$ \\
\hline \multicolumn{2}{|c|}{ Dual variables } \\
\hline$\alpha_{s, t, k}$ & $\begin{array}{l}\text { Dual variable associated to the storage content of storage unit } s \text { at time step } \\
t \text { in scenario } k \text {. }\end{array}$ \\
\hline $\bar{\lambda}_{s, t, k}, \underline{\lambda}_{s, t, k}$ & $\begin{array}{l}\text { Dual variables associated to upper and lower limits of the charging capacity } \\
\text { storage unit } s \text { at time step } t \text { in scenario } k \text {. }\end{array}$ \\
\hline $\bar{\mu}_{s, t, k}, \underline{\mu}_{s, t, k}$ & $\begin{array}{l}\text { Dual variables associated to upper and lower limits of discharging storage } \\
\text { unit } s \text { at time step } t \text { in scenario } k \text {. }\end{array}$ \\
\hline$\pi_{t, k}$ & Electricity market clearing prices at time step $t$ in scenario $k(€ / \mathrm{MWh})$. \\
\hline
\end{tabular}

\section{Introduction}

Power systems are undergoing an accelerated energy transition, in part driven by environmental concerns, with the vast deployment and integration of variable generation from renewable energy sources (RES) being one of the most remarkable changes. However, high shares of variable renewable energy sources (VRES) come along with a number of operational and planning challenges. Energy Storage Systems (ESS) may offer the needed flexibility to cost-efficiently integrate renewable generation. This has motivated ample research in their applications, benefits and new potential services and products in electricity and ancillary service markets (EASAC, 2017; IEC, 2011). Relevant literature comprises business models and revenue streams for ESS (He et al., 2011), the role of storage to accommodate high shares of VRES (Taylor et al., 2013; Su and El Gamal, 2013; Yang and Yang, 2019; de Sisternes et al., 2016: McPherson et al., 2018), the importance of adequately siting and sizing ESS (Dvorkin et al., 2017; Pandžić et al., 2018), its interaction with transmission switching (Peker et al., 2018), the relevance of ownership structures (Taylor, 2015; Hartwig and Kockar, 2016, Koirala et al., 2018), participation in reserve markets (Akhavan-Hejazi and Mohsenian-Rad, 2014; Pandžić et al., 2018; Schillemans et al. 2018) and its impact on system-wide emissions (Lin et al., 2016), the real-time dispatch of storage under uncertainty (Papavasiliou et al., 2018) and leveraging distributed ESS via aggregators (Contreras-Ocaña et al., 2017).

The value of energy storage has been acknowledged by policy makers and regulators around the world, who have taken steps to bring grid-scale storage back to the agenda. For example, the European Commission has included energy storage among the priority actions of the Strategic Energy Technology Plan (European Commission, 2017). The State of California has set targets to procure $1325 \mathrm{MW}$ utility-owned energy storage to be completed 
by 2020 (CAISO, 2014) and in Australia the installation of the largest grid-scale battery (100 MW lithium-ion battery) in December 2017 paved the way for more large-scale storage projects (Hutchins, 2018).

Although continued research efforts and technological developments lead to decreasing costs of ESS (IRENA, 2017b; Nykvist and Nilsson, 2015), substantial changes may be required to current market designs to ensure market prices provide adequate signals to invest in, optimally operate and properly remunerate ESS for the services they provide (IRENA, 2017a). Only few power systems, mainly in the US, have recognized ESS as particular assets with certain characteristics that set them apart from the traditional entities participating in the wholesale market (PJM, 2017; CAISO, 2018, 2007). The European Commission (2018) has recently given a final definition for energy storage, which is currently picked up in the design of European electricity markets and regulation (see further and EPEX SPOT SE (2018)).

In liberalized electricity markets, bid structure sets the limits and means for, i.a., generating companies, load serving entities and energy storage system owners to communicate their offers to the market for every trading interval. Thus, bid structures delimit the playing field for market players to strategically participate in the market. In the light of the energy transition, conventional bid structures defined as price-quantity pairs may have to be revised to allow reflecting the operational constraints of all resources, including ESS (Pérez Arriaga and Knittel et al, 2016).

Even though there is extensive scientific literature that addresses the operational benefits of ESS for power systems and the economic implications of its strategic behavior (see above and Section 2), there are few scientific papers that deal with bid structures as instruments facilitating the strategic participation of ESS in electricity markets. In the scientific literature, the decision to adopt a particular bid structure is usually predefined or based on the market design of the studied power system. However, a comprehensive study assessing the impact of different bid structures on, e.g., the profitability of ESS is missing.

This paper intends to fill this gap and constitutes a first step in establishing a common ground to compare and analyze bid structures from the perspective of a storage system owner. We study the implications of different market designs, in particular bid structures, on the value of a strategically operated storage in a high RES system. This is analyzed within the context of energy-only day-ahead electricity markets where storage owners profit from price arbitrage and face uncertainties due to the limitedly predictable availability of VRES in the market.

In light of these challenges, we formulate four bilevel optimization problems, representing the strategic participation of a single price-making agent, i.e., the merchant ESS operator, in a pool electricity market. The objective of the ESS owner is to maximize its operating profit, taking into account its operational constraints and the allowed bid structures. Four distinct bid structures (i.e., quantity-based, price-based, price-quantity-based and complex bids) are investigated. In a case study, we demonstrate that when the bidder can perfectly anticipate the impact of its decisions on the market dispatch and prices (i.e., deterministic anticipation on the market outcome), both the profitability of a strategic ESS owner and system performance (e.g., social welfare) are indifferent to the bid structure adopted. Nevertheless, when 
the bidder is uncertain of market outcomes (i.e., stochastic anticipation), the bid structure has an impact on the ESS owner's bids, profitability, operation and, consequently, albeit to a lesser extent, the overall system's performance.

The main contribution of this paper is a comparative analysis of the implications of bid structures in electricity markets on the profitability and bid behavior of strategic ESS owners. We focus on the treatment of ESS-specific technical constraints within the bids, the effect of uncertainty on market outcomes and relevant policy implications. This analysis is based on bilevel optimization problems, which are designed to reflect the participation of strategic ESS owners in stylized electricity markets under different bid structures. Our models may be used by (1) storage owners to support their bidding strategies under different market designs and (2) by policy makers, regulators and market operators in the assessment of different market designs and to detect, e.g., market power.

This paper is organized as follows. Section 2 provides an overview of bid structures found in electricity markets worldwide, as well as relevant scientific literature studying the strategic participation of ESS owners and generating companies in these markets. Second, we present our methodology and the mathematical formulation of the bilevel optimization problems, used to study the ESS owner's strategic participation in pool-based electricity markets (Section 3). Third, a case study is introduced (Section 4). Before concluding (Section 6), we discuss the implications of our work in Section 5.

\section{Literature review}

First, we introduce the four bid structures studied in this paper. Second, we link them to bid structures found in electricity markets around the world. Last, we position our methodology (Section 3) w.r.t. state-of-the-art modeling approaches.

\subsection{Bid structures studied in this paper}

Current electricity market designs are characterized by a variety of bid structures that in turn restrict the way agents strategically participate and operate their assets. We propose a bid structure classification based on (i) the decision variables of the ESS owner and communicated to the market operator in the form of bids (e.g., quantity, price or both) and (ii) the inclusion of the ESS's operational constraints in the market clearing procedure (i.e., simple or complex bids). We distinguish between four bid structures (Table 1), based on different bid structures found in current electricity markets and scientific literature:

1. Simple quantity bidding: The ESS participates in the market by deciding availability offers (i.e., charging and discharging capacity) in the form of quantity bids, offered to the market at predetermined prices (cost-based approach). Consequently, the ESS's strategic behavior is limited to withhold capacity in order to shape prices to increase profits. Note that the ESS owner is not required to withhold capacity, but may do so if it increases its operating profit. This type of bid structure is in use in cost-based markets throughout Central and South America (e.g., Brazil (Hammons et al., 2002) and Mexico (Secretaria de Energia - SENER, 2015)). 


\begin{tabular}{cc|c|c}
\hline \hline & & \multicolumn{2}{|c}{ Type of bid } \\
& & Quantity-based bid & Price-based bid \\
\hline \begin{tabular}{c} 
Detail of $\begin{array}{c}\text { Nisclosed } \\
\text { technical } \\
\text { information }\end{array}$ \\
\cline { 2 - 4 }
\end{tabular} & No disclosure & $\begin{array}{r}\text { 1. Simple quantity bidding } \\
\text { Full disclosure }\end{array}$ & \multicolumn{2}{|c}{$\begin{array}{c}\text { 2. Simple price-quantity pairs bidding } \\
\text { 4. Complex bidding }\end{array}$} \\
\hline \hline
\end{tabular}

Table 1. Categorization of the four bid structures structures studied in this paper according to the type of bid (price/quantity bid) and the amount of technical information disclosed to the market operator.

2. Simple price bidding: The ESS makes all its discharging and charging capacity available at self-determined prices, reflecting its willingness to charge and discharge at each time step (price-based approach). Although this type of bid structure is - to the best of the author's knowledge - not in use in any electricity market, it has been studied by, e.g., Ruiz and Conejo (2009); Shahmohammadi et al. (2018a b) and Zou et al. (2016)

3. Quantity-price pairs bidding: This bidding structure represents a combination of the previous two approaches. The strategic agent is able to withhold charging/discharging capacity and to express its willingness to charge/discharge in the form of price bids. Price-quantity bids are found in many electricity markets around the world, including the European EPEX spot market (EPEX SPOT, 2014), ERCOT (Electric Reliability Council of Texas, 2016) and CAISO (Mohsenian-Rad, 2016) in the US and Austrialia's AEMO (Australian Energy Market Operator, 2010).

4. Complex bidding: The strategic agent discloses all its technical constraints to the market operator. The market operator is responsible for fulfilling these constraints when clearing the market. Similar to the first case, we limit the strategic behavior under this bidding structure to withholding capacity (i.e., quantity-based). Note that price-based and price-quantity complex bidding structures are also possible. Examples of complex bid structures are found in PJM (PJM, 2017), CAISO (CAISO, 2007) and EPEX Spot (EPEX SPOT SE, 2018).

Note that the first three bid structures have in common that the technical constraints specific to the energy storage system, and in particular the constraints related to the energy balance of the storage unit, are the responsibility of the ESS owner. In the complex bid structure, the market operator is responsible to fulfill these technical constraints and to maintain the energy balance of the storage system when clearing the market. This implies that the market operator has knowledge of the ESS's characteristics, technical constraints and of the value of the stored energy. As we will discuss in Section5, the ESS's characteristics disclosed to the market operator may differ from the true technical constraints of the storage, however, for the sake of a fair comparison with other bid structures, we assume the ESS owner's bids correspond to the real technical constraints of the ESS. In what follows, we will more extensively discuss (i) examples of electricity markets where these bid structures are in use and (ii) scientific papers adopting specific bid structures. 


\subsection{Bid structures in existing electricity markets}

Electricity markets based on simple quantity bids limit the participation of the agents to bid the availability of their assets (i.e., quantity) at a cost-based economic offer (including opportunity costs), which are regulated and audited (Munoz et al., 2017). This cost-based market is implemented in most hydro-thermal systems in Latin-America (e.g., Brazil and Mexico (Hammons et al., 2002; Secretaria de Energia - SENER, 2015)). Under such a market structure, a ESS owner would bid separately its availability to discharge (as any other generation unit) and its availability to charge (as any other load). Scientific literature adopting this bid structure include, i.a., Nasrolahpour et al. (2016); Pandžić and Kuzle (2015); Pandžić et al. (2019); Cui et al. (2018); Dvorkin et al. (2018); Govaerts et al. (2018) and Mohsenian-Rad (2016).

Even though a simple price bid structure is, to the best of the authors' knowledge, not implemented in practice in any electricity market, it is included in this paper to depict (i) the difficulty in incorporating technical constraints within this bid structure and (ii) that these bids allow communicating the value of the energy stored. Simple price bids are studied by, e.g., Ruiz and Conejo (2009); Shahmohammadi et al. (2018a. b); Zou et al. (2016).

Simple price-quantity pairs is a bid structure found in most electricity markets, i.a., in Europe. This structure allows storage owners send price-quantity bids to charge and discharge, which would be treated as demand and generation bids separately, similar to the simple quantity bids. For example, in Spain market players have to register either as supply or load entities in order to participate in the wholesale market (Store Project, 2014). This means that ESS must register and send separate bids as a generator and as a consumer in the form of price-quantity pairs. In the eyes of the market operator, these are separate bids, which can be cleared independently. Consequently, the ESS owner is responsible to keep the desired balance in its storage system. In the EPEX spot market, covering Germany, France, United Kingdom, the Netherlands, Belgium, Austria, Switzerland and Luxembourg, bidders are allowed to use linked price-quantity pair bids which are orders that intend to incorporate technical and economical constraints in the bids (EPEX SPOT, 2014). The electricity market design in Texas (Electric Reliability Council of Texas, 2016) and Australia also allow simple price-quantity bids (Australian Energy Market Operator, 2010). Scientific literature adopting this bid structure includes Hartwig and Kockar (2016); Vespermann et al. (2017); Pandžić et al. (2019); Jia et al. (2018); Kardakos et al. (2016); Chen et al. (2019) and Mohsenian-Rad (2016).

In a complex quantity bid structure the market operator recognizes storage systems as a different type of resource whose charging (load) and discharging (supply) bids are associated to the same market player. Electricity markets employing this bid structure include, i.a., PJM and CAISO. In PJM, the operation of the system and clearing prices are based on a Security Constrained Economic Dispatch problem considering the technical constraints of the transmission network, conventional generators and pumped hydro storage units (PJM, 2017). Pumped hydro storage systems have to bid minimum and maximum generation and pumping capacities, reservoir limits and efficiency factors. With this information, the ISO incorporates a conservation flow constraint that assures that pumping will only occur if the cost of pumping is offset by an associated benefit of generating in subsequent periods. 
Similarly, the Californian ISO, CAISO, established a new type of resource, Non-Generator Resources (NGR), which can operate either as generator or load and are constrained in terms of energy (MWh) (CAISO, 2018). The NGR provide capacity and energy limits to the ISO, which are enforced through the State of Charge (SOC) constraint in the market and operation. The NGR are able to choose between two types of bids: (i) self-schedule bids which do not include a price component, meaning that the buyer (seller) is willing to buy (sell) electricity regardless of the price (i.e., inelastic demand); and (ii) economic bids that includes a price component (i.e., willingness to buy/sell electricity) (CAISO, 2007). At the end of 2018, the European Power Exchange EPEX SPOT introduced loop block orders, which allows bundling buy and sell block orders on the European day-ahead market EPEX SPOT SE (2018). As such loop block orders must be jointly rejected or accepted, they may be used to reflect the bids of a ESS owner. Scientific literature adopting complex quantity bid structures include, i.a., Huang et al. (2018); Pandžić et al. (2018); Schillemans et al. (2018) and Ye et al. (2017).

\subsection{Modeling the strategic participation of Energy Storage Systems in electricity markets}

The scientific literature dealing with the participation of ESS in electricity markets is split into three broad categories based on the perspective of storage operating decisions and its impact on market prices: (i) system models, capturing the operation of ESS in a centralized cost-minimization problem, (ii) optimization or mixed complementary problems, representing price-taking ESS owners and (iii) mathematical or equilibrium problems with equilibrium constraints, studying price-making ESS owners.

Often, researchers adopt a centralized system approach in which ESS are dispatched to maximize social welfare and/or enhance the security of power systems (Parvania et al., 2014; Papavasiliou et al., 2018; Su and El Gamal, 2013; Chen et al., 2012; Bruninx et al., 2016). Even though a centralized approach provides valuable insights in the operation and benefits of ESS, it is critical to bear in mind that most power systems are governed by marketbased principles and therefore individual (i.e., market player) objectives must be taken into account 1 .

The optimal operation and offering strategy of a price-taker ESS is determined based on an expected price profile or set of price profiles. For example, Akhavan-Hejazi and Mohsenian-Rad (2014) study the optimal operation of storage in day-ahead energy and reserve markets. Contreras-Ocaña et al. (2017) consider the relationship between aggregators and their constituent storage units and their effect on system welfare. Scheduling centralized and distributed EES systems in day-ahead electricity markets is the topic at hand in Parvania et al. (2014). He et al. (2016) incorporate cycling costs into a profit maximization model to determine the optimal bids in day-ahead energy, spinning reserve, and regulation markets. The models employed in the aforementioned papers share the common assumption of an invariant price profile governing the operation or bid strategy of the ESS.

\footnotetext{
${ }^{1}$ Note that, in a perfectly competitive environment, minimizing the system costs in a convex economic dispatch formulation also yields to the maximum profit for the ESS owners (Castillo and Gayme, 2013).
} 
However, the potential influence of large volumes of grid-scale storage and those particular situations in which ESS's behavior could have an impact on electricity prices, such as during peak load hours or when grid constraints are binding, raise questions regarding their participation in markets and the implications of different market designs. Researchers investigating the strategic behavior of a price-making ESS in electricity markets typically adopt one of the following three approaches: (i) the impact on prices is determined by means of a demand function (Schill and Kemfert, 2011; Baillo et al., 2004; De La Torre et al., 2002) or market resilience functions (Brijs et al., 2016); (ii) the strategic behavior is represented through a bilevel optimization model or mathematical problem with equilibrium constraints (MPEC), reflecting a Stackelberg relationship between the ESS owner and the market (operator) (Hartwig and Kockar, 2016; Mohsenian-Rad, 2016; Ye et al., 2016, 2017; Nasrolahpour et al., 2017; Schillemans et al., 2018; Cui et al., 2018; Dvorkin et al., 2018; Pandžić et al., 2018; Jia et al., 2018; Chen et al., 2019; Govaerts et al., 2018; Kardakos et al., 2016; Huang et al., 2018) or (iii) multiple strategic agents are considered in an equilibrium problem with equilibrium constraints (EPEC) (Sioshansi, 2014; Shahmohammadi et al., 2018b a; Pandžić et al. 2019; Zou et al., 2016). This last type of problems can be considered as a set of MPECs with a common set of equilibrium constraints. In bilevel problems, the upperlevel (UL) problem constitutes a profit maximization of the strategic agent, i.e. the ESS owner, deciding on bids, considering the optimal response of the lower-level (LL) problem, representing a market clearing defining the dispatch and corresponding market prices.

The aforementioned MPECs and EPECs are employed to study a variety of relevant problems in the context of the participation of energy storage in electricity markets. Schillemans et al. (2018), e.g., study the participation of ESS in joint energy, operating reserve and balancing markets, similar to the topic at hand in Nasrolahpour et al. (2017). Cui et al. (2018) and Hartwig and Kockar (2016) investigate the strategic participation of an ESS owner in transmission system-constrained electricity markets. Ye et al. (2016) focus on how strategic energy storage owners may limit the market power of conventional generators. In another paper, Ye et al. (2017) study how ESS owners may withhold capacity to maximize their profits in day-ahead electricity markets. Govaerts et al. (2018) study the impact of distribution tariffs on the attainable operating profit of a strategic aggregator of distributed battery storage systems. Similarly, Jia et al. (2018), Mohsenian-Rad (2016) and Kardakos et al. (2016) develop a bilevel optimization model governing the strategic participation of an aggregator in day-ahead electricity and operating reserve markets. Pandžić et al. (2019) focus on the interaction of multiple battery storage aggregators in day-ahead electricity markets, which they study via an EPEC. Coordinating transmission system and energy storage investments is the topic at hand in Dvorkin et al. (2018) and Pandžić et al. (2018). Dvorkin et al. (2017) and Chen et al. (2019) study the siting and sizing problem faced by ESS investors. Zou et al. (2016) study the different equilibria that may arise in pool electricity markets, considering various types of storage units and their interaction with wind power generation. Shahmohammadi et al. (2018b a) describe the effect of storage on the attainable surplus in energy markets and the storage owner's profit via an EPEC.

Regardless of the approach or market participation issue studied, a common threat of these works is that their authors emphasize that strategic ESS owners tend to under-utilize 
their capacity in imperfectly competitive markets compared to perfectly competitive markets in order to maintain the price differences between discharging and charging hours, thereby increasing their profitability. This finding not only has welfare implications, but also prevents to unlock the full potential/flexibility of storage systems. However, the analysis in the papers above typically consider a single bid structure, which does not allow assessing the impact of such bid structures in the utilization of ESS and system performance. Pandžić et al. (2019) form an exception to this observation, as they study simple quantity and simple pricequantity bids. However, they (1) do not consider simple price bids and complex quantity bids and (2) perform their analysis assuming perfect foresight on the market outcomes. To capture the aforementioned effects, and to study the impact of the assumed bid structure hereon, we propose four stochastic bilevel optimization problems - one for each bid structure - in Section 3 .

\section{Methodology}

The proposed models assume there is a single price-making agent (leader), owning a ESS, that participates in a pool electricity market (follower) to maximize its profit. The resulting bilevel model, reflecting a Stackelberg Game (Hobbs, 1999), consists of an upper-level (UL) problem, representing the ESS profit optimization problem, and a lower-level (LL) problem simulating the market clearing outcome in which the market operator maximizes social welfare. The ESS owner defines an optimal offering strategy, considering the optimal response of the market operator in terms of dispatch and prices, and the allowed bid structures. This bilevel approach allows endogenously determining both the ESS owner's offering strategy and the resulting market clearing prices. The proposed bilevel optimization problems are similar to those found in the scientific literature (see, i.a. Hartwig and Kockar (2016); Mohsenian-Rad (2016); Ye et al. (2016, 2017); Nasrolahpour et al. (2017); Schillemans et al. (2018); Cui et al. (2018); Dvorkin et al. (2018); Pandžić et al. (2018); Jia et al. (2018); Chen et al. (2019); Govaerts et al. (2018); Kardakos et al. (2016) and Huang et al. (2018)).

Since the LL problem is continuous and convex (see below), it can replaced by its KarushKuhn-Tucker (KKT) optimality conditions which are incorporated in the UL problem as constraints, resulting in a Mathematical Problem with Equilibrium Constraints or MPEC (Hobbs et al., 2000). After reformulating some non-linear expressions using the Big-M method (Fortuny-Amat and McCarl, 1981) and the strong duality theorem, inspired by Ruiz and Conejo (2009), the problem can be reformulated as an equivalent Mixed-Integer Linear Programming (MILP) problem. In what follows, we first discuss the assumptions made during the model development. Second, the bilevel optimization problems are introduced. For the sake of clarity and conciseness, the derivation of the KKT conditions and the equivalent MILP problem is not included in the main body of the text. The interested reader is referred to Appendix A.

\subsection{Assumptions}

The presented models comprise an energy-only day-ahead market with hourly bids. Network constraints are omitted in order not to pollute the analysis with excessive market power 
of the ESS owner induced by transmission congestion (Borenstein et al., 1999). We consider two cases w.r.t. the ESS owner's foresight on the available RES in the market, hence, market outcomes (see Section 4). In a first, deterministic case, the ESS owner has perfect foresight on the available RES. In the second, stochastic case, the uncertainty is represented by introducing a set of scenarios representing the available energy from renewable sources offered in the market, which have an impact on clearing prices. The strategic agent has to decide upon a set of bids common to all scenarios that maximizes its expected profit, considering all the possible price and dispatch scenarios (i.e., lower-level clearing problems). The non-strategic agents are assumed to be price-takers and participate in the market by offering all their generation capacity at variable generation cost. Maintenance costs of the ESS are not considered. A cyclical boundary condition enforces that the energy stored in the ESS at the end of the horizon is equal to the initial storage level, ensuring the comparability of results.

\subsection{Mathematical formulation}

Recall that we will consider four bid structures (Table 1): (i) simple quantity bidding; (ii) simple price bidding; (iii) simple quantity-price pairs bidding; and (iv) complex quantity bidding. Contrary to the fourth bid structure, the first three approaches have in common that the technical constraints of the storage units are taken care of by the ESS owner (i.e., enforced in the UL problem) and they are not considered when clearing the market (i.e., in the LL problem). In fact, the simple quantity bidding and simple price bidding cases can be seen as simplified versions of the simple quantity-price pairs bidding model. Therefore, we will first present the bilevel optimization model considering simple quantity-price pair bids as a reference. The required changes in the formulation to reflect simple quantity bid and simple price bid structures are described in Section 3.2.2 and 3.2.3. The bilevel optimization problem describing the ESS owner's profit maximizing problem under the assumption of complex bid structures is presented in Section 3.2.4. The differences between the models are summarized in Table 2 .

\subsubsection{Simple quantity-price pair bidding}

In the upper-level (UL) problem, the strategic ESS owner maximizes its expected operating profit, which depends on the lower level market clearing prices $\pi_{t, k}$ and accepted charge $r_{s, t, k}$ and discharge $q_{s, t, k}$ bids (Eq. (1)). The technical constraints of the ESS comprise the lower and upper bounds of energy stored (Eq. (2)), the dynamic variation of energy levels in storage due to charging and discharging decisions affected by their corresponding efficiency (Eq. (3)) and the cyclical constraint stating that the energy content at the end of the horizon must be equal to the initial period (Eq. (4)). Constraints (5)-(8) limit the quantities and prices bid into the market.

$$
\max _{x, y, \beta^{q}, \beta^{r}} \sum_{s \in S} \sum_{t \in T} \sum_{k \in K} p_{k} \cdot \pi_{t, k}\left(q_{s, t, k}-r_{s, t, k}\right)
$$


Table 2. Impact of the bid structures on the upper level and lower level problems. Indices of variables are suppressed for the sake of readability.

\begin{tabular}{|c|c|c|c|c|}
\hline & Simple quantity & Simple price & $\begin{array}{l}\text { Price-quantity } \\
\text { pairs }\end{array}$ & $\begin{array}{l}\text { Complex quan- } \\
\text { tity }\end{array}$ \\
\hline $\begin{array}{l}\text { Decision vari- } \\
\text { ables in UL } \\
\text { problem }\end{array}$ & $\begin{array}{l}\text { Availability } \\
(x, y)\end{array}$ & $\begin{array}{l}\text { Willingness to } \\
\text { charge/discharge } \\
\left(\beta^{q}, \beta^{r}\right)\end{array}$ & $\begin{array}{l}\text { Availability } \\
(x, y) \& \text { will- } \\
\text { ingness to } \\
\text { charge/discharge } \\
\left(\beta^{q}, \beta^{r}\right)\end{array}$ & $\begin{array}{l}\text { Availability } \\
(x, y)\end{array}$ \\
\hline $\begin{array}{l}\text { Impact of bids } \\
\text { on } L L \text { problem }\end{array}$ & $\begin{array}{l}\text { Upper bounds } \\
\text { of charg- } \\
\text { ing/discharging } \\
\text { quantities }\end{array}$ & $\begin{array}{l}\text { Objective func- } \\
\text { tion }\end{array}$ & $\begin{array}{l}\text { Upper bounds } \\
\text { of charg- } \\
\text { ing/discharging } \\
\text { quantities } \\
\& \quad \text { objective } \\
\text { function }\end{array}$ & $\begin{array}{l}\text { Upper bounds } \\
\text { of charg- } \\
\text { ing/discharging } \\
\text { quantities }\end{array}$ \\
\hline $\begin{array}{l}\text { Responsible to } \\
\text { maintain the } \\
\text { balance on the } \\
\text { ESS }\end{array}$ & $\begin{array}{l}\text { ESS's } \\
\text { (UL) }\end{array}$ & $\begin{array}{l}\text { ESS's owner } \\
\text { (UL) }\end{array}$ & $\begin{array}{l}\text { ESS's } \quad \text { owner } \\
(\mathrm{UL})\end{array}$ & $\begin{array}{l}\text { Market Opera- } \\
\text { tor (LL) }\end{array}$ \\
\hline
\end{tabular}

subject to

$$
\begin{aligned}
& \underline{v}_{s} \leq v_{s, t, k} \leq \bar{v}_{s} \quad \forall s \in S, \forall t \in T, \forall k \in K \\
& v_{s, t, k}=v_{s, t-1, k}+\delta_{s}^{c} r_{s, t, k}-1 / \delta_{s}^{d} q_{s, t, k} \quad \forall s \in S, \forall t \in T, \forall k \in K \\
& v_{s, t=0, k}=v_{s, t=T, k} \quad \forall s \in S, \forall k \in K \\
& 0 \leq x_{s, t} \leq \bar{q}_{s} \quad \forall s \in S, \forall t \in T \\
& 0 \leq y_{s, t} \leq \bar{r}_{s} \quad \forall s \in S, \forall t \in T \\
& 0 \leq \beta_{s, t}^{q} \leq \Pi \quad \forall s \in S, \forall t \in T \\
& 0 \leq \beta_{s, t}^{r} \leq \Pi \quad \forall s \in S, \forall t \in T \\
& q_{s, t, k}, r_{s, t, k}, \pi_{t, k}=\operatorname{argmin} \mathcal{L}_{k}\left(x_{s, t}, y_{s, t}, \beta_{s, t}^{q}, \beta_{s, t}^{r}\right) \quad \forall k \in K
\end{aligned}
$$

Based on commonly used linear cost-minimization dispatch models and adapted from the lower-level (LL) model presented by Hartwig and Kockar (2016), LL problem (10)-(16) represents the day-ahead market clearing problem in each scenario $k$ (set $K$ ):

$$
\left\{\min \mathcal{L}_{k}\left(x_{s, t}, y_{s, t}, \beta_{s, t}^{q}, \beta_{s, t}^{r}\right)=\min \sum_{t \in T}\left(\sum_{s \in S} \beta_{s, t}^{q} \cdot q_{s, t, k}-\sum_{s \in S} \beta_{s, t}^{r} \cdot r_{s, t, k}+\sum_{i \in I} c_{i, t} \cdot g_{i, t, k}+c^{\phi} \cdot \phi_{t, k}\right)\right.
$$


subject to

$$
\begin{aligned}
& \sum_{i \in I} g_{i, t, k}+\sum_{j \in J} w_{j, t, k}+\sum_{s \in S} q_{s, t, k}-\sum_{s \in S} r_{s, t, k}+\phi_{t, k}=D_{t} \quad:\left[\pi_{t, k}\right] ; \forall t \in T \\
& 0 \leq q_{s, t, k} \leq x_{s, t} \quad:\left[\underline{\mu}_{t, k}, \bar{\mu}_{t, k}\right] ; \quad \forall s \in S, \forall t \in T \\
& 0 \leq r_{s, t, k} \leq y_{s, t} \quad:\left[\underline{\lambda}_{t, k}, \bar{\lambda}_{t, k}\right] ; \quad \forall s \in S, \forall t \in T \\
& 0 \leq w_{j, t, k} \leq \bar{w}_{j, t, k} \quad \forall j \in J, \forall t \in T \\
& 0 \leq g_{i, t, k} \leq \bar{g}_{i} \quad \forall i \in I, \forall t \in T \\
& \left.0 \leq \phi_{t, k} \leq D_{t} \quad \forall t \in T \quad\right\} \quad \forall k \in K
\end{aligned}
$$

The market operator maximizes the total surplus, which is equivalent to minimizing the total operating costs assuming an inelastic demand (Eq. (10)). Equation (11) imposes the power balance at every time step $t$. The associated dual variable $\pi_{t, k}$ corresponds to the market clearing price. Constraints (12)-(13) limit the cleared charging and discharging quantities to the bids of the ESS owner. The dispatch of renewable energy sources is limited to their forecasted availability $\bar{w}_{j, t, k}$ in each scenario (Eq. (14)). Constraints (15) and (16) define the power generation limits of non-strategic conventional generation $g_{i, t, k}$ and load shedding $\phi_{t, k}$. Note that the ESS owner's bids are not scenario dependent.

\subsubsection{Simple quantity bidding}

Quantity bids are included the UL problem as decision variables and passed on to the LL problem as upper bounds (i.e., parameters) on the amount of energy the market operator is allowed to charge or discharge from the storage unit (12)-(13). These capacities are bid into the market at fixed prices, here assumed equal to the price cap (17) and floor (18). Charging bids are associated with a willingness to charge equal to the price cap, which guarantees that the market operator will accept this bid. Similarly, discharging bids are associated with a price-bid equal to zero, ensuring discharging bids are cleared.

$$
\begin{array}{ll}
\beta_{s, t}^{r}=\Pi & \forall s \in S, \forall t \in T \\
\beta_{s, t}^{q}=0 & \forall s \in S, \forall t \in T
\end{array}
$$

\subsubsection{Simple price bidding}

The price bids are decision variables of the UL problem and reflected in the objective function of LL as parameters representing additional costs and revenues to the market operator $(10)$. The storage owner offers all its capacity at these endogenously determined prices, $\beta^{r}$ and $\beta^{q}$, to the market. Compared to the formulation presented in Eq. (1)-(16), this bid structure requires modifications in constraints (5)-(6):

$$
\begin{array}{ll}
x_{s, t}=\bar{q}_{s} & \forall s \in S, \forall t \in T \\
y_{s, t}=\bar{r}_{s} & \forall s \in S, \forall t \in T
\end{array}
$$




\subsubsection{Complex Bidding}

Under the assumption of complex quantity bidding, the market operator is responsible for satisfying the technical constraints of the ESS when clearing the market. This implies that, besides bidding availability such as in simple quantity structure, the strategic agent must disclose technical information to the market, including charging/discharging capacity limits, efficiency, energy storage limits. This is reflected in the bilevel optimization problem by moving the technical constraints of the ESS from the UL problem to the LL problem (Eq. (32)-(34)):

In the upper level problem, the ESS owner decides on its charge/discharge bids in order to maximize its expected operating profit, given its anticipation on the market clearing prices $\pi_{t, k}:$

$$
\max _{x, y} \sum_{s \in S} \sum_{t \in T} \sum_{k \in K} p_{k} \cdot \pi_{t, k}\left(q_{s, t, k}-r_{s, t, k}\right)
$$

subject to

$$
\begin{aligned}
& 0 \leq x_{s, t} \leq \bar{q}_{s} \quad \forall s \in S, \forall t \in T \\
& 0 \leq y_{s, t} \leq \bar{r}_{s} \quad \forall s \in S, \forall t \in T \\
& q_{s, t, k}, r_{s, t, k}, \pi_{t, k}=\operatorname{argmin} \mathcal{L}_{k}\left(x_{s, t}, y_{s, t}\right) \quad \forall k \in K
\end{aligned}
$$

Lower level problem (25)-(34) reflects the market clearing problem considering complex bids for each scenario $k$ (set $K$ ) (formulation adapted from the LL problem presented in (Huang et al. 2018)). Note that the objective of the market operator (Eq. (25)) no longer contains terms associated with the ESS system. Indeed, in the studied complex bidding paradigm, the market operator endogenously values stored energy, therefore no longer requiring the ESS to express an explicit willingness to charge/discharge:

$$
\left\{\min \mathcal{L}_{k}\left(x_{s, t}, y_{s, t}\right)=\min \sum_{t \in T}\left(\sum_{i \in I} c_{i, t} \cdot g_{i, t, k}+c^{\phi} \cdot \phi_{t, k}\right)\right.
$$

subject to

$$
\begin{aligned}
& \sum_{i \in I} g_{i, t, k}+\sum_{j \in J} w_{j, t, k}+\sum_{s \in S} q_{s, t, k}-\sum_{s \in S} r_{s, t, k}+\phi_{t, k}=D_{t} \quad:\left[\pi_{t, k}\right] ; \quad \forall t \in T \\
& 0 \leq q_{s, t, k} \leq x_{s, t} \quad:\left[\underline{\mu}_{t, k}, \bar{\mu}_{t, k}\right] ; \quad \forall s \in S, \forall t \in T \\
& 0 \leq r_{s, t, k} \leq y_{s, t} \quad:\left[\underline{\lambda}_{t, k}, \bar{\lambda}_{t, k}\right] ; \quad \forall s \in S, \forall t \in T \\
& 0 \leq g_{i, t, k} \leq \bar{g}_{i} \quad \forall i \in I, \forall t \in T \\
& 0 \leq w_{j, t, k} \leq \bar{w}_{j, t, k} \quad \forall j \in J, \forall t \in T \\
& 0 \leq \phi_{t, k} \leq D_{t} \quad \forall t \in T \\
& \underline{v}_{s} \leq v_{s, t, k} \leq \bar{v}_{s} \quad \forall s \in S, \forall t \in T \\
& v_{s, t, k}=v_{s, t-1, k}+\delta_{s}^{c} r_{s, t, k}-1 / \delta_{s}^{d} q_{s, t, k} \quad:\left[\alpha_{s, t, k}\right] ; \quad \forall s \in S, \forall t \in T \\
& \left.v_{s, t=0, k}=v_{s, t=T, k} \quad \forall s \in S \quad\right\} \quad \forall k \in K
\end{aligned}
$$


Table 3. Capacity and generation costs of the conventional and renewable units in the test system.

\begin{tabular}{c|c|c}
\hline Generator & $\begin{array}{c}\text { Installed capacity } \\
(\mathrm{MW})\end{array}$ & $\begin{array}{c}\text { Generation costs } \\
(€ / \mathrm{MWh})\end{array}$ \\
\hline Unit 1 & 500 & 10 \\
Unit 2 & 500 & 10 \\
Unit 3 & 300 & 15 \\
Unit 4 & 300 & 20 \\
Unit 5 & 300 & 55 \\
Unit 6 & 300 & 60 \\
Unit 7 & 100 & 80 \\
Unit 8 & 100 & 95 \\
Wind & 400 & - \\
Solar & 450 & - \\
\hline \hline
\end{tabular}

\section{Case Study}

We study the impact of different bid structures on the bidding strategy of a ESS owner on a specific day in a fictitious power system. The lowest demand occurs during hour 4 (1530 MW) and the peak load occurs in hour 20 (2400 MW). All conventional and renewable generators (Table 3) are assumed to be owned by non-strategic agents. Additionally, the system includes a ESS, owned by the strategic agent, of $400 \mathrm{MW}$ of charging and discharging power capacity and 1600 MWh of storage capacity. For sake of simplicity, we set the charging and discharging efficiency to 1 . Note that this does not impact the qualitative discussion of our results, nor does it affect the complexity of the decision problems faced by the storage operator or market operator.

As a benchmark, we simulate the operation of ESS in a perfect competitive environment, represented as a centralized economic dispatch problem (Parvania et al., 2014). In this centralized approach, the market operator has control over the ESS, which will be employed to minimize system costs by charging it during periods of low electricity generation costs and discharge it during peak price periods.

We analyze the implications of different bidding structures on the strategy of a storage owner, the agent's profitability, storage operation and social welfare. We differentiate between a deterministic case, i.e., a case in which the ESS owner has perfect foresight on the market outcome, and a stochastic case, in which the ESS owner is unsure of the market clearing prices due to uncertainty on the RES-based generation offered in the market. In the deterministic case, the energy available from RES is calculated as the average of the set of renewable generation profiles considered in the stochastic case. This scenario set was obtained by randomly selecting daily generation profiles from the data registered by the Belgian TSO Elia (Elia NV, 2018) ${ }^{2}$ The generation profiles were normalized and scaled according to the solar and wind capacity in the power system at hand (Table 3).

\footnotetext{
${ }^{2}$ If one aims to capture the uncertainty on, e.g., wind and solar power forecasts, dedicated scenario
} 
The presented case study does not focus on quantifying, e.g., the potential profitability of a strategic storage owner under different bid structures, but to provide insight in the relevance of market design and its relation with the strategic behavior of an ESS owner.

\subsection{Strategic bidding with perfect foresight}

In a deterministic bilevel optimization context, the strategic storage owner has the ability to shape the desired clearing prices and quantities via its bids in order to maximize its profit, regardless of the allowed bid structure. Indeed, in the bilevel optimization structure, the ESS owner assumes that the dispatch of the storage is prioritized and optimally modulated in order guarantee its technical feasible operation and to maximize the ESS owner's profits. This optimistic outcome is embedded in the Stackelberg Game between the market operator (follower) and the ESS owner (leader), which assumes the market operator reacts optimally (from the perspective of the ESS owner) to the actions of the leader. As a result, one observes (i) similar market outcomes (prices and quantities) and storage operation and (ii) the same storage owner's profit under each bid structure. Note that Huang et al. (2018) also report this equivalence between market outcomes under complex quantity bids and a 'deregulated market mechanism', i.e., when the ESS owner decides directly on the operation of the ESS, under the assumption of perfect foresight.

Contrary to a centralized approach where the operation of storage results in flattening price profiles by fully charging during low price periods and fully discharging during peak prices, the price-making ESS owner will send bids that maintains the difference between market prices during discharging and charging periods to maximize its profits. All the bid structures enable the ESS owner to exert strategic behavior and raise its profit from $€ 88,000$ assuming a centralized operation of storage to $€ 182,000$ in a deterministic, strategic bidding approach, regardless of the considered bid structure.

In what follows, we expose how these results arise under different bid structures. For sake of brevity, we do not provide detailed numerical results, but focus on the underlying mechanisms linking the bid structure, the bilevel optimization problem and market outcomes.

In the case of a simple quantity bid structure, the charging bids are considered as an additional inelastic demand and the discharging bids are always infra-marginal (i.e., willingness to discharge equal to zero), thereby guaranteeing that the bids sent by the storage owner are always accepted. This characteristic enables the storage owner to obtain an optimal bidding strategy that, by strategically withholding discharging/charging capacity, shapes its dispatch and prevailing market prices in order to maximize its profits and fulfills technical constraints.

To maximize its profit under the simple price bid structure, the ESS owner provides price bids, both for charging and discharging, that coincide to the anticipated market prices, which

generation and reduction techniques are available (Bruninx, 2016, Bruninx and Delarue, 2016). In this paper, however, we do not attempt to capture the characteristics of a specific source of uncertainty, but strive to understand how different bid structures affect the ability of ESS owners to participate in energy markets, considering uncertain market outcomes. For sake of simplicity, we therefore selected the scenarios at random from historical data, provided by the Belgian TSO Elia (Elia NV, 2018). 
coincide with the variable cost of the expected non-strategic marginal unit. In a deterministic setting, offering the entire storage capacity at the expected market prices makes the ESS the marginal unit at every time step. In a bilevel optimization problem, the ESS owner assumes that the dispatch of the storage is prioritized and optimally modulated in order guarantee its technical feasible operation and to maximize the ESS owner's profits. Note, however, that in reality the market operator decides the dispatch of the marginal units indistinctly of the bidders (e.g., through a pro-rata methodology), which may lead to deviating profits and technical feasibility issues, threatening the applicability of simple price bids. Indeed, simple price bids are unable to incorporate information on state-of-charge or capacity limits in the bid. This structure, therefore, cannot guarantee that the resulting dispatch satisfies the feasible operation of storage, e.g., the energy balance constraint of the storage might be violated. We will return to these issues in Section 5. It is worth noting that these issues do not arise in the simple quantity bid structure.

The third bid structure, price-quantity pairs, yields price bids similar to the simple price bids, i.e., equal to the expected market price. However, this bid structure endows the storage owner with a greater degree of physical control of the dispatch of the storage by deciding on the quantity offered to the market. Indeed, the storage operator's offered quantity may be lower than the capacity of the storage system, thereby explicitly withholding capacity, e.g., for other purposes or to maximize its profits on the energy market. In our analysis, the offered quantities match the cleared quantities exactly, which guarantees that its technical constraints are always satisfied and profits are maximized.

While the simple bids require that the value of the stored energy - which ultimately determines the dispatch of storage in the market - is implicitly incorporated in quantity bids or explicitly declared through price bids by the storage operator, the complex bidding structure allows valuing stored energy within the market. Indeed, the dynamics and constraints of the ESS are explicitly represented in the market clearing, hence, the market operator not only guarantees that the technical constraints are fulfilled but also acknowledges the value of (dis)charging and its impact on subsequent periods. Contrary to simple bids, this allows explicit inter-temporal links in the value of stored energy. Therefore, the complex bid structure does not require to incorporate the charging quantity bids as inelastic demand, nor do the bids need to match the cleared quantities. However, by strategically withholding capacity, the ESS owner may ensure that its profit is maximized.

\subsection{Strategic bidding under uncertainty}

The model incorporates uncertainty in the ESS owner's anticipation of the market clearing outcome via a set of possible scenarios of RE generators' bids that lead to different market clearing outcomes, and consequently affect the strategy of the storage owner. To illustrate how this type of uncertainty impacts the bids of the ESS owner, we consider a set of 5 equiprobable, randomly selected scenarios $(|K|=5)$ (see above).

Before we discuss the impact of the allowed bid structure on the decision problem of the ESS owner in detail, we provide a high-level summary of the impact of these bid structures 
Table 4. Summary of the results for strategic bidding under uncertainty: the estimated generation costs, as a metric for the efficiency of the resulting dispatch schedule, the ESS owner's profit and the difference in surplus for each market actor w.r.t. the surplus observed in the centralized dispatch case. Results are expressed in thousands of euros $(\mathrm{k} €)$.

\begin{tabular}{l|l|l|l|l|l}
\hline \hline & $\begin{array}{l}\text { Centralized } \\
\text { dispatch }\end{array}$ & $\begin{array}{l}\text { Quantity } \\
\text { bids }\end{array}$ & Price bids & $\begin{array}{l}\text { Price- } \\
\text { quantity } \\
\text { bids }\end{array}$ & $\begin{array}{l}\text { Complex } \\
\text { bids }\end{array}$ \\
\hline $\begin{array}{l}\text { Generation } \\
\text { costs }\end{array}$ & 915 & 917 & 918 & 916 & 915 \\
$\begin{array}{l}\text { ESS owner's } \\
\text { profit }\end{array}$ & 8.8 & 15.8 & 16.1 & 18.1 & 18.2 \\
\hline $\begin{array}{l}\Delta \text { Consumer } \\
\text { surplus }\end{array}$ & - & 27.2 & 118.8 & 79.6 & 23.7 \\
$\begin{array}{l}\Delta \quad \text { Genera- } \\
\text { tor's surplus } \\
\Delta \quad- \\
\text { owner's ESS } \\
\text { surplus }\end{array}$ & - & -36.2 & -128.5 & -90.1 & -33.4 \\
$\Delta$ Total sur- \\
plus
\end{tabular}

on the surplus of each of the actors in the market (Table 4 ) $3^{3}$ In contrast to the deterministic case, the bid structure now affects ESS owner's strategy, profitability and system performance. The strategic behavior of the ESS owner always leads to inefficiencies from a social welfare perspective w.r.t. the centralized dispatch benchmark, as indicated by the

${ }^{3}$ We calculate the surplus for each of the actors as follows:

- The generators surplus is defined as the difference between the clearing price and the cost of generation, multiplied with the cleared generation of each unit at each time step:

$$
\sum_{k \in K} \sum_{t \in T} \sum_{i \in I} p_{k} \cdot\left(\pi_{t, k}-c_{i, t}\right) \cdot g_{i, t, k}
$$

- The consumers surplus is calculated as the difference between the utility of demand and the clearing price, multiplied with the cleared demand:

$$
\sum_{k \in K} \sum_{t \in T} p_{k} \cdot\left(c^{\phi}-\pi_{t, k}\right) \cdot\left(D_{t}-\phi_{t, k}\right)
$$

- The ESS owners welfare is calculated as the ESS owners profit:

$$
\sum_{k \in K} \sum_{t \in T} \sum_{s \in S} p_{k} \cdot \pi_{t, k} \cdot\left(q_{s, t, k}-r_{s, t, k}\right)
$$

The total surplus is calculated as the sum of the three surplus components above. 
negative change in total surplus and elevated generation costs. This observation is in line with the results of the analytical model developed by Sioshansi $(2014)$.

The complex bid structure allows the storage operator to obtain the highest profit, hence, surplus while keeping the market outcome as closest to the central dispatch benchmark. This is the result of the endogenous consideration of the value of stored energy in the market clearing problem and the transfer of responsibility to satisfy technical constraints to the market operator, as we will discuss extensively below. Simple bid structures entail lower profits for the ESS owner, higher generation costs and lower total surplus. The conventional generators bear the loss in surplus, whereas consumers benefit, confirming the conclusion of Sioshansi (2014).

Note furthermore that higher operating profits for the ESS owner, which may be interpreted as a metric for market power, do not necessarily imply higher generation costs and total surplus losses, which may be considered a proxy for market efficiency. Indeed, the complex bid structure and, to a lesser extent, the price-quantity pair bid structure allow the storage operator to offer more capacity to the market, which has a positive impact on their operating profit and the generation costs. In what follows, we discuss how the differences in operating profit and generation costs arise under the different bid structures.

The simple quantity bidding (Figure 1a $1 \mathrm{~b}$ ) guarantees that, regardless of the market clearing scenario $k$, the total charging/discharging capacity offered to the market is accepted. In consequence, the actual dispatch of the storage for all the scenarios exactly follows the profile defined by its charging and discharging bids (Figure 1b). Since satisfying the technical constraints is the responsibility of the storage owner, the ESS owner not only withholds storage capacity for strategic purposes (i.e., to affect prices), but also to guarantee that the technical constraints are fulfilled in all possible scenarios. This leads to low operating profits (compared to results under other bid structures) and an increase in generation costs, as less storage capacity is available to the market operator to perform arbitrage to lower generation costs.

Mathematically, this is enforced via stationary condition A.34): $-\Pi+\pi_{t, k}-\underline{\lambda}_{s, t, k}+\bar{\lambda}_{s, t, k}=$ 0 . Dual variables $\underline{\lambda}, \bar{\lambda}$ are associated to the lower and upper constraints limiting the charging of storage between zero and the quantity bid $y$. Given that the price cap $\Pi$ is a constant and $\Pi \gg \pi_{t, k}$, the equation above holds only when $\bar{\lambda}$ is greater than zero. Given the complementary slackness condition A.44, this dual variable can be different from zero only when the upper bound constraint of the charging variable is binding (i.e., $y_{s, t}=r_{s, t, k}$ ), forcing the actual dispatch in all the scenarios to be equal to the upper limit defined by the quantity bid. A similar analysis can be done for discharging bids.

In the case of simple price bid structure (Figure 1c 1d), the strategic agent will try to match the highest (lowest) market clearing price across scenarios with its discharging (charging) bid. This ensures bids are either not accepted or accepted as the marginal unit, in which case the market operator is assumed to clear the market optimally from the perspective the storage owner. This bid structure thus tolerates dispatch schedules that differ across scenarios (Fig. 1d), in contrast to the simple quantity bid structure (Fig. 1b), which leads to higher expected operational profits while guaranteeing that the technical 
constraints of the storage system are met (given the assumption of an optimistic solution to the lower level, embedded in the bilevel optimization problem structure). Note that despite the greater operational flexibility available to the market operator (Fig. 1d), higher generation costs are observed under this bid structure. The implications of the assumption of the optimal market reaction on the applicability of this bid structure and our results are discussed in Section 5 .

The interaction between price bids, clearing prices and storage operation in a simple price bid setting is governed by stationary conditions (A.33): $\beta_{s, t}^{q}-\pi_{t, k}-\underline{\mu}_{s, t, k}+\bar{\mu}_{s, t, k}=0$ and A.34): $-\beta_{s, t}^{r}+\pi_{t, k}-\underline{\lambda}_{s, t, k}+\bar{\lambda}_{s, t, k}=0$. These equations show that a positive market price $\left(\pi_{t, k}\right)$ can only be offset either by a charging/discharging price bid of the same magnitude or by binding charging/discharging capacity constraints in order to allow their corresponding dual variables to be different from zero (underpinned by the corresponding complementary slackness conditions A.38, A.39, A.43), A.44). In order to modulate the ESS operation and keep the stored energy within technical limits, the ESS owner will always send price bids that do not lead to be fully dispatched, i.e., simple price bids that are either not accepted or accepted as the marginal unit considering all the scenarios. As a consequence, the charging and discharging price bids result in lower and upper bounds, respectively, of the clearing prices in the different scenarios (Figure 1c).

In contrast, the greater bidding flexibility of simple price-quantity bid structure (Figure $1 \mathrm{~h}-1 \mathrm{~g})$ allows the strategic agent to define price bids that not necessarily match the expected price scenarios. However, during some periods it is optimal, from the ESS owner's perspective, to send price bids that match the bid of the marginal unit in all scenarios (e.g., charging bids in hours 2 to 5). In those hours, the dispatch of the storage is governed by the market operator, who is assumed to respond optimally to these price bids. During other parts of the day, the quantity bids provide hard limits to the storage's operation, which allows ensuring feasibility of the dispatch schedule. For instance, in $t=6$ the charging price bid matches the expected market price of all the scenarios except for scenario $k=1$, which is considerably lower. Consequently, while in scenarios 2 to 5 the ESS is the marginal unit and its dispatch is decided by the market operator, the ESS is infra-marginal in scenario 1, but the storage owner is able to set a limit on its dispatch by means of the quantity component of the bid. Hence, this bid structure tolerates scenario-specific storage dispatch schedules (as in the simple price bids) and, in addition, allows the storage operator to set strategic limits to the available capacity through the quantity component of the bid structure. As a result, the storage operator is able to secure higher operating profits (compared to the quantity bids and price bids). Furthermore, this also leads to lower generation costs, as more storage capacity is available to the market operator to perform arbitrage (Fig. 1f]).

Similarly, the complex quantity bidding benefits both the system and the storage owner (Table 4). The market operator is responsible for satisfying the technical constraints associated to the storage, enforced in each scenario $k$, which implies that the market operator links the charging and discharging bids to the same unit in each scenario $k$. Indeed, the market operator is informed on the impact of its dispatch decisions in the state-of-charge of the storage system. In turn, this allows the storage owner to offer more capacity than under 
simple bid structures, in which the storage owner must ensure feasibility of the dispatch schedule across scenarios, and allows the market operator to make use of more of the available storage capacity to minimize system costs. Note that optimally scheduling the storage system and linking charge and discharge bids implies a valuation of stored energy by the market operator (see below). The ESS owner still exhibits strategic behavior by withholding capacity in order to maximize its profits, but typically provides more capacity to the market (e.g., compare Figure $1 \mathrm{~h}$ and Figure 1b). This results in the highest operating profit for the storage owner and generation costs close to those under a centralized dispatch paradigm.

The explicit valuation of stored energy by the market operator is mathematically illustrated by the stationary conditions A.46): $-\pi_{t, k}-\underline{\mu}_{s, t, k}+\bar{\mu}_{s, t, k}-1 / \delta_{s}^{d} \cdot \alpha_{s, t, k}=0$; and A.47): $\pi_{t, k}-\underline{\lambda}_{s, t, k}+\bar{\lambda}_{s, t, k}+\delta_{s}^{c} \cdot \alpha_{s, t, k}=0 . \alpha$ is the dual variable of the energy balance constraint and thus - by definition - the economic value of stored energy. These equations ensure an explicit relationship between the value of storage, the market price the storage operation decisions. Note that $\alpha_{s, t, k}$ is not present in the stationary conditions of the other bid structures (see Section 3 and Appendix A.

In contrast to simple bids, the explicit, endogenous valuation of stored energy within the market, possible with complex bids, informs the market operator of the consequences of charging or discharging the ESS. Moreover, this bid structure tolerates scenario-specific dispatch schedules, which enables the market operator to perform arbitrage to lower generation costs while guaranteeing that technical constraints of storage are met in every scenario (Fig. 1h). 


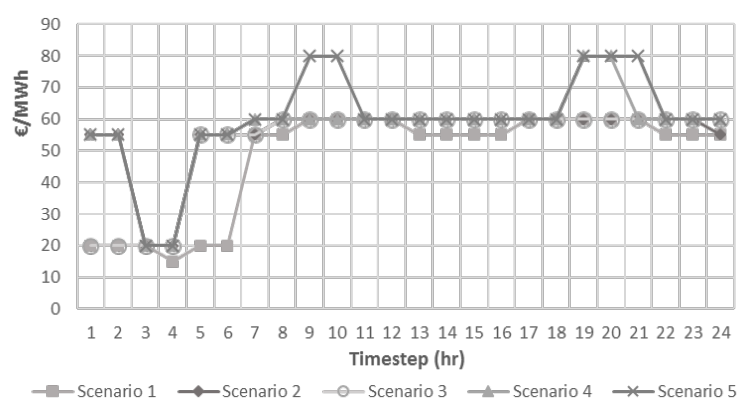

(a) Expected clearing prices with Simple quantity bid structures

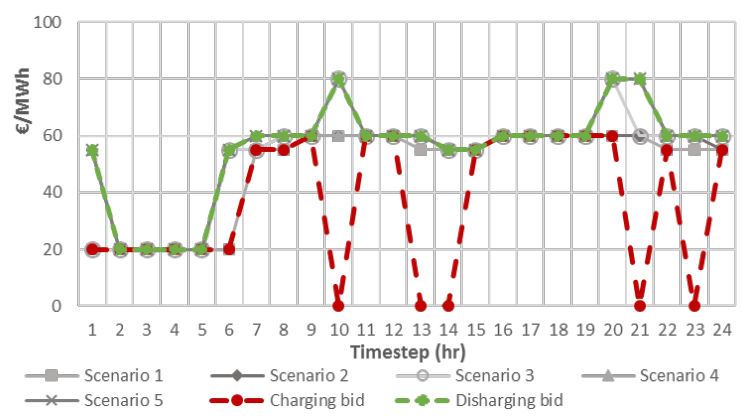

(c) Price-bid profile and expected clearing prices with Simple price bid structures

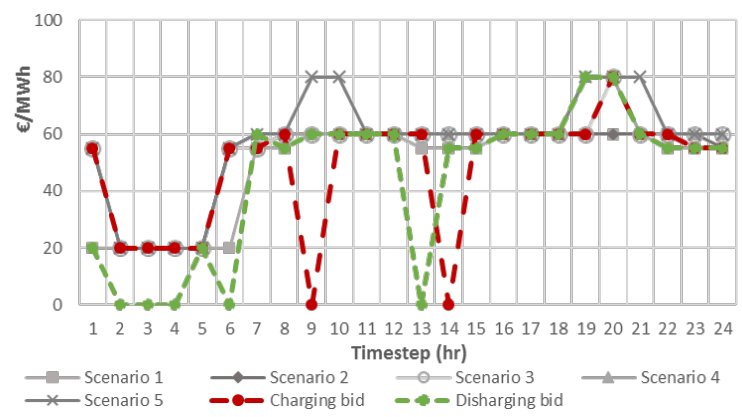

(e) Price-bid profile and expected clearing prices with Simple price-quantity bid structures

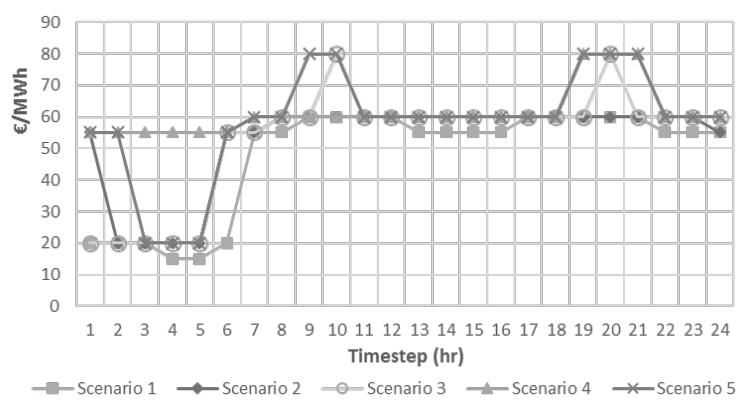

(g) Expected clearing prices with Complex quantity bid structures

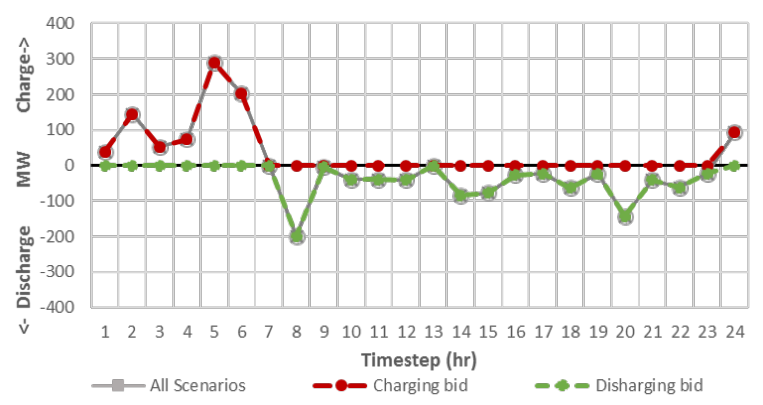

(b) Quantity-bid profile and operation of ESS with Simple quantity bid structures

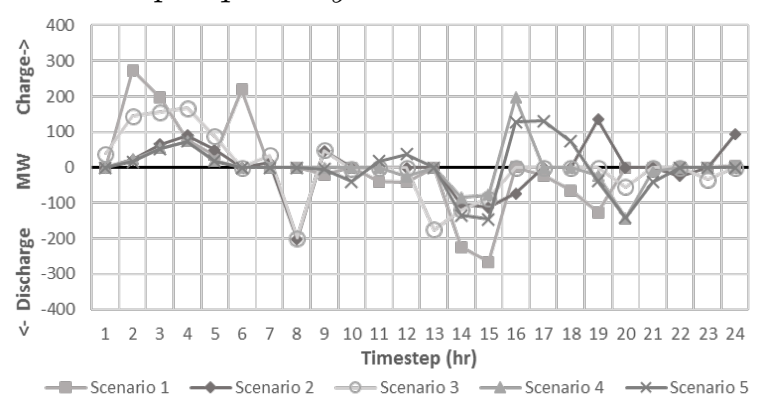

(d) Operation of storage responding to Simple price bids structures

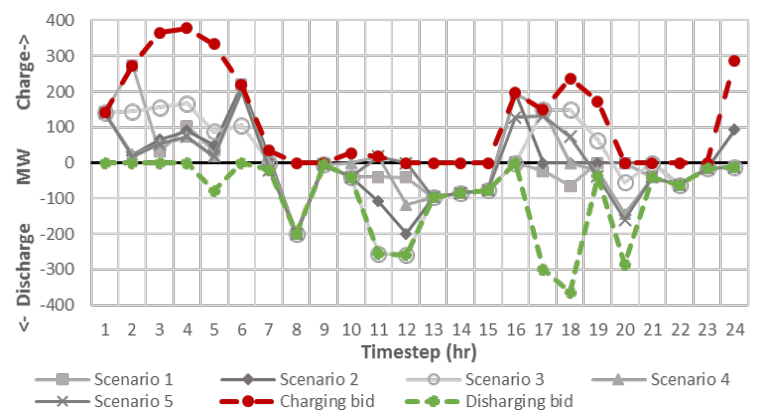

(f) Quantity-bid profile and operation of ESS with Simple price-quantity bid structures

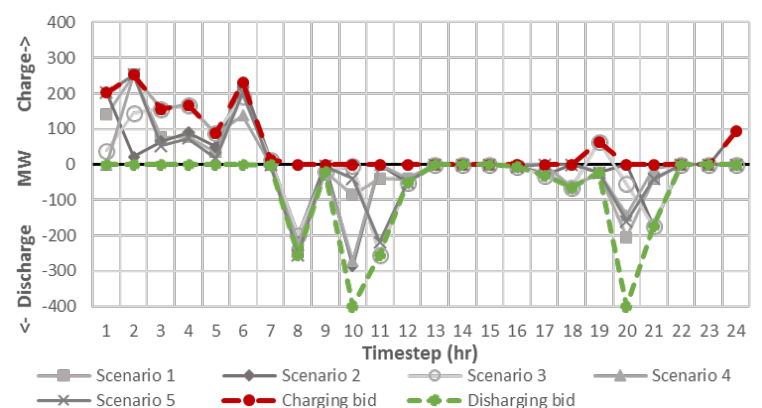

(h) Quantity-bid profile and operation of ESS with Complex quantity bid structures

Figure 1. Storage operation and price profiles under a Simple quantity bid, Simple price bid, Simple price-quantity bid and Complex quantity bid structure. 


\section{Discussion}

This section provides some insight in the implications of different bid structures, which may be distilled from the analysis presented in the previous sections. Section 5.1 illustrates that, despite the fact that the deterministic approach leads to the same results in all bid structures, different bids provide different means to exert the strategic behavior and to guarantee feasibility of the dispatch schedule of the ESS. Subsequently, we discuss the effect of uncertainty on the ESS owner's strategy under different bid structures (Section 5.2) and other competition concerns (Section 5.3).

\subsection{Strategic behavior \& feasibility}

The participation of any agent in a market is motivated by its expectation to make a profit and is limited by its technical constraints and the bid structure in place, which defines the boundary conditions of the agent's strategy. In order to obtain both economically efficient and technically feasible market outcomes, most market designs allow, to some extent, incorporating the technical constraints of conventional generation assets (e.g., ramp rates, minimum up/down times or minimum output) through designated bid structures. However, this is typically not the case for ESS, because the limited storage energy content is not captured by any of the simple-bid structures commonly found in real-life markets.

In the case of simple quantity-based structures, the ESS owner behaves strategically by withholding capacity in order to affect market prices favorably. As elaborated in Section 4. the submitted simple quantity bids define the operating schedule of the storage, which must be feasible in the considered market outcome. Consequently, withholding capacity is the only way a ESS owner may (i) exhibit strategic behavior to affect prices and (ii) keep the balance in the energy stored and fulfill its technical constraints. This leads to overly conservative bidding behavior.

Similarly, strategic behavior and feasibility of the ESS's dispatch are also blended together in a simple price bid structure. The ESS owner will submit bids that attempt to match clearing prices ${ }^{4}$. However, there is no guarantee that the actual dispatch and prevailing market prices will maximize its profits, and more importantly, do not violate its storage content-related constraints. Simple price bids are, by definition, unable to capture the timecoupling nature of the storage operation (i.e., link discharge and charge bids), threatening the applicability of simple price bids in real markets.

Since simple price-quantity pair bidding is a combination of the previous two structures, it provides storage owners with the means to account for their energy-content related constraints (as with simple quantity bids). The price-component in the bid allows the ESS to set the price as a marginal generation or load unit, which may be reflected in greater profits. Even though simple price-quantity pair bids provide more flexibility in the offering strategy to the ESS owner, the optimistic assumption w.r.t. the prevailing dispatch and prices as for

\footnotetext{
${ }^{4}$ By definition, the bilevel model structure implies that the ESS owner takes an optimistic position with respect to the market clearing outcome: if the bid price matches the market clearing price, the ESS owner assumes it will be accepted and the ESS will be optimally dispatched.
} 
the simple price bid still holds in the simple price-quantity pair bid structure, which may also lead to an overestimation of the profitability. Although the actual dispatch when the price component of the bid is marginal may not lead to a desired dispatch, the quantity component of the bids may be used to ensure feasibility. In turn, despite the greater flexibility, this bid structure may still lead to conservative bidding strategies.

In contrast, the complex bid structure allows ESS owners to decouple the feasibility of the resulting dispatch and strategic behavior: while the strategic behavior is exerted by withholding capacity, the feasibility of the ESS's operating schedule is guaranteed by the market operator, who dispatches the available storage capacity to maximize social welfare while maintaining the stored energy content within the technical limits. This, however, does require to declare information on the efficiency and storage capacity of the ESS to the market operator. Note that the apparent capacity communicated to the market may not correspond to the true technical capacity, e.g., when the ESS owner reserves some storage capacity to participate in other markets or to balance its own portfolio (see below), and that this information is not revealed to other market participants. In addition, placing the responsibility to fulfill the technical constraints with the market operator allows innovative forms of market participation, such as a revenue-constrained bid or an extension to complex price-quantity pairs.

\subsection{The impact of uncertainty}

In the simple quantity bid structure, the ESS owner must ensure feasible operation of the ESS operating schedule across all possible market outcomes. As all bids may be accepted, this results in an overly conservative bidding strategy. In turn, this leads to under-utilization of the ESS at the expense of lower operating profits for the ESS owner and higher system costs (Table 4). Note, however, that this implies that the feasibility of the operating schedule of the ESS is guaranteed, even for market outcomes not considered in the ESS owner's bid problem.

Under the simple price bid structure, the ESS owner matches extremes of the market clearing prices with its charging and discharging price bids (Figure 1c). The actual operation of the ESS in different scenarios, however, corresponds to an 'optimistic' dispatch decided by the market operator in order to maximize ESS's profits and satisfy its technical constraints in all the scenarios (see, e.g., Figure $1 \mathrm{c}$ and $1 \mathrm{~d}$ in time steps $t=16-19$ ). As the number of scenarios and, hence, the number of possible market clearing prices considered increases, the share of scenarios in which the price bids match the market clearing prices typically decreases. Consequently, those bids end up being a binary decision to be fully dispatched (i.e., when the clearing price is higher (lower) than the charging (discharging) price bid) or not dispatched at all in most scenarios. This constitutes significant risks to the strategic agent in terms of profitability and feasibility of the resulting operating schedule. Indeed, in market outcomes not considered in the ESS owner's bid problem, the ESS may be required to follow an operating schedule that would violate the technical constraints of the ESS system. The ESS owner will hence not be able to fulfill the operating schedule as determined by the market. To avoid this, risk-averse ESS owners may submit overly conservative bids, 
which would lead to lower ESS profits and higher system costs. This limits the real-world applicability of simple price bid structures as a tool to stimulate ESS market participation.

In the quantity-price pairs bid structure the price component allows (i) fine-tuning the operation of storage in the scenario(s) when the price-bid matches the clearing price and (ii) determining the operation mode (i.e. whether to charge, discharge or none) when the price bid is greater or lower than the market price. The quantity component allows, in addition to strategically withholding capacity, ensuring that the storage content remains within the technical limits across all market outcomes (see above). The price-bid component will lose relevance as the number of distinct market scenarios considered in the ESS owner's problem increases, since it is more difficult to match the clearing prices in a larger set of scenarios. Hence, the larger scenario sets considered in the ESS owner's problem are, characterizing highly uncertain market outcomes, the closer the behavior and results of the quantity-price pairs bid structure are to the simple quantity bid paradigm.

While in simple bid structures both feasibility concerns and the strategic behavior were tangled within the bid and therefore both were affected by uncertainty, the complex bid structure decouples feasibility, guaranteed in all scenarios by the market operator, and strategic behavior. This provides greater flexibility in allowed bids, which resulted not only in greater benefits to the storage owner but also in system performance (e.g., lower system operating costs) (Table 4). The lack of valuation of stored energy by the market operator in simple bid structures prevents to use of storage assets efficiently in correspondence to systems needs. With simple bid structures, the market operator will use the storage given the ESS owners expected need of the system for such storage reflected by the ESS owner's anticipation on market clearing prices. Hence, the efficiency in the operation of storage responding to system's needs depends on how accurate the ESS owner can anticipate the system's needs for flexibility through price signals. In contrast, the complex bid structure allows the market operator to value stored energy by acknowledging the inter-temporal links between bids. Consequently, the market operator may make use of available storage capacity in correspondence to the actual system needs for such flexibility.

\subsection{Competition concerns \& compatibility with other markets}

Bid structures encompass market design aspects beyond the quantitative assessment of system performance and strategic behavior of the agents. For instance, bid structures also have implications on how markets are organized, on competition and compatibility with other markets.

A particular advantage of quantity-based bidding structures is that they allow the strategic agent to explicitly reserve part of its capacity, e.g., to participate in other markets or to balance their own portfolio.

Providing more information to the market operator (e.g., under the form of complex bids) leads to higher utilization rates of the ESS, and consequently, higher operating profits for the ESS owner and lower system operating costs. However, the full disclosure of technical information and transferring of responsibility to fulfill storage's technical constraints to the market operator has a number of implications. 
Firstly, contrary to the simple bidding structures where the ESS owner can decide its own dispatch following the bids profile, in complex bidding the market operator decides the dispatch of storage for different market outcomes. Consequently, the ESS owner has less information on the state of the storage system at each time step before the clearing of the market, which lowers its autonomy to operate the ESS and the information available to the storage owner on its own asset to participate in other markets.

Secondly, disclosing technical information (only to the market operator) such as the amount of energy stored at every period in time and the charging and discharging efficiency of the storage can be seen as a threat to the strategic position and autonomy of the storage owner. However, disclosing this information could also be seen as a step towards a more transparent market and it can be used by, e.g., regulators to oversee the market.

\section{Conclusion}

The flexibility provided by ESS may play a pivotal role in the transition towards power systems with high shares of renewable generation. This paper intends to analyze the relevance and implications of bid structures as a market design feature to steer the strategic participation of ESS owners in electricity markets. We formulate four bilevel optimization problems to represent the strategic participation of a price-making ESS owner under different bid structures (i.e., quantity-based, price-based, price-quantity-based and complex bids).

The extent to which the bid structure in place allows incorporating the technical constraints of the ESS, e.g., the limited energy storage content, sets boundaries to the strategic participation of the ESS owner, its profitability and the performance of the system. With increasing uncertainty on the market outcome, ESS owners are likely to adopt excessively conservative behavior under simple bid structures because they should incorporate the inherent 'risk' of not satisfying storage's technical constraints. By transferring the responsibility of fulfilling technical constraints to the market (i.e. complex bids), this conservative behavior may be avoided and enable to unlock the potential/flexibility of storage systems, not only for their owners but also for the system. The complex bid structure enables a valuation of storage within the market, allowing the market operator to use the storage capacity available in the market in correspondence to system needs under different market outcomes. However, the disclosure of technical information and the transfer of responsibilities from the storage owner to the market operator, limits the information on the future state of the storage system available to the ESS owner and lowers its autonomy to operate the storage.

This work may be strengthened in the following ways. First, the interaction with other markets, such as reserves and balancing markets might be considered. Second, other sources of uncertainty such as the bid prices and quantities of the conventional generators may be included. Another relevant extension is the consideration of strategic behavior from other market players. Third, in the context of high RES systems, the models presented here can be extended to execute an assessment of the strategic behavior of agents with a portfolio of ESS and variable renewable assets in order to provide insights in the portofolio value of ESS. Fourth, the transfer of responsibilities to the market operator to fulfill ESS' 
technical constraints has relevant implications in terms of market regulation, responsibilities and liabilities of market authorities, which merit further research. Last, considering other demand characteristics, as well as other power systems, could enhance the numerical analysis in this paper.

\section{Acknowledgment}

K. Bruninx is a post-doctoral research fellow of the Research Foundation - Flanders (FWO) and the Flemish Institute for Technological Research (VITO).

\section{Bibliography}

Akhavan-Hejazi, H., Mohsenian-Rad, H., 2014. Optimal operation of independent storage systems in energy and reserve markets with high wind penetration. IEEE Transactions on Smart Grid 5 (2), 1088-1097.

Australian Energy Market Operator, 2010. AN INTRODUCTION TO AUSTRALIA'S NATIONAL ELECTRICITY MARKET. Tech. rep., Australian Energy Market Operator, Melbourne, Australia.

Baillo, A., Ventosa, M., Rivier, M., Ramos, A., 2004. Optimal offering strategies for generation companies operating in electricity spot markets. IEEE Transactions on Power Systems 19 (2), 745-753.

Borenstein, S., Bushnell, J., Knittel, C. R., 1999. Market power in electricity markets: Beyond concentration measures. The Energy Journal 20 (4), 65-88.

Brijs, T., Geth, F., Siddiqui, S., Hobbs, B. F., Belmans, R., 2016. Price-based unit commitment electricity storage arbitrage with piecewise linear price-effects. Journal of Energy Storage 7, 52-62.

Bruninx, K., 2016. Improved modeling of unit commitment decisions under uncertainty. Phd dissertation, KU Leuven, Leuven, Belgium.

Bruninx, K., Delarue, E., 2016. Scenario reduction techniques and solution stability for stochastic unit commitment problems. In: IEEE International Energy Conference (ENERGYCON). Leuven, Belgium, pp. 1-7.

Bruninx, K., Dvorkin, Y., Delarue, E., Pandžić, H., Dhaeseleer, W., Kirschen, D. S., 2016. Coupling pumped hydro energy storage with unit commitment. IEEE Transactions on Sustainable Energy 7 (2), 786-796.

CAISO, 2007. CALIFORNIA INDEPENDENT SYSTEM OPERATOR CORPORATION FERC ELECTRIC TARIFF AMENDED AND RESTATED THIRD REPLACEMENT VOLUME NO. I Substitute Original Sheet No. 380. Tech. rep., CAISO, Folsom, CA, USA.

CAISO, 2014. Advancing Maximizing Value of Energy Storage Technology - California Roadmap. Tech. rep., CAISO, Folsom, CA, USA.

CAISO, 2018. Non-Generator Resource (NGR) and Regulation Energy Management (REM) Overview Phase 1. Tech. rep., CAISO, Folsom, CA, USA.

Castillo, A., Gayme, D. F., 2013. Profit maximizing storage aladdress in power grids. In: Proceedings of the IEEE Conference on Decision and Control. Florence, Italy, pp. 429-435.

Chen, D., Jing, Z., Tan, H., 2019. Optimal siting and sizing of used battery energy storage based on accelerating benders decomposition. IEEE Access (Early Access), 1-10.

Chen, S. X., Gooi, H. B., Wang, M. Q., 2012. Sizing of energy storage for microgrids. IEEE Transactions on Smart Grid 3 (1), 142-151.

Contreras-Ocaña, J. E., Ortega-Vazquez, M. A., Zhang, B., 2017. Participation of an Energy Storage Aggregator in Electricity Markets. IEEE Transaction on Smart Grid 10 (2), 1171-1183.

Cui, H., Li, F., Fang, X., Chen, H., Wang, H., April 2018. Bilevel arbitrage potential evaluation for gridscale energy storage considering wind power and lmp smoothing effect. IEEE Transactions on Sustainable Energy 9 (2), 707-718.

De La Torre, S., Arroyo, J. M., Conejo, A. J., Contreras, J., 2002. Price maker self-scheduling in a poolbased electricity market: A mixed-integer LP approach. IEEE Transactions on Power Systems 17 (4), 1037-1042. 
de Sisternes, F. J., Jenkins, J. D., Botterud, A., 2016. The value of energy storage in decarbonizing the electricity sector. Applied Energy 175, $368-379$.

Dvorkin, Y., Fernandez-Blanco, R., Kirschen, D. S., Pandžić, H., Watson, J. P., Silva-Monroy, C. A., 2017. Ensuring Profitability of Energy Storage. IEEE Transactions on Power Systems 32 (1), 611-623.

Dvorkin, Y., Fernndez-Blanco, R., Wang, Y., Xu, B., Kirschen, D. S., Pandi, H., Watson, J., Silva-Monroy, C. A., Jan 2018. Co-planning of investments in transmission and merchant energy storage. IEEE Transactions on Power Systems 33 (1), 245-256.

EASAC, 2017. Valuing dedicated storage in electricity grids. Tech. rep., EASAC, Brussels, Belgium.

Electric Reliability Council of Texas, 2016. ERCOT Market Education - Basic Training Program. Tech. rep., ERCOT, Austin, TX, USA.

Elia NV, 2018. Grid data. Available online: www.elia.be/en/grid-data.

EPEX SPOT, 2014. Introduction of Smart block bids - Linked block orders and Exclusive block orders. Tech. rep., EPEX SPOT, Brussels, Belgium.

EPEX SPOT SE, 2018. Epex spot introduces curtailable blocks and loop blocks on all day-ahead markets. Press release. Available online: https://www.epexspot.com/en/press-media/press/details/press/ EPEX_SPOT_introduces_curtailable_blocks_and_loop_blocks_on_all_Day-Ahead_markets.

European Commission, 2017. Energy storage - the role of electricity. Tech. rep., European Commission, Brussels, Belgium.

European Commission, 2018. Energy Storage. Tech. rep., European Commission, Brussels, Belgium.

Fortuny-Amat, J., McCarl, B., 1981. A representation and economic interpretation of a two-level programming problem. JORS 32 (9), 783-792.

Govaerts, N., Bruninx, K., Delarue, E., 2018. Impact of distribution tariff design on the profitability of aggregators of distributed energy storage systems. In: 15th International Conference on the European Energy Market (EEM). pp. 1-5.

Hammons, T., Rudnick, H., Barroso, L. A., 2002. Latin America: Deregulation in a Hydro-Dominated Market. HRW, 1-5.

Hartwig, K., Kockar, I., 2016. Impact of Strategic Behavior and Ownership of Energy Storage on Provision of Flexibility. IEEE Transactions on Sustainable Energy 7 (2), 744-754.

He, G., Chen, Q., Kang, C., Pinson, P., Xia, Q., 2016. Optimal Bidding Strategy of Battery Storage in Power Markets Considering Performance-Based Regulation and Battery Cycle Life. IEEE Transactions on Smart Grid 7 (5), 2359-2367.

He, X., Delarue, E., D'haeseleer, W., Glachant, J. M., 2011. A novel business model for aggregating the values of electricity storage. Energy Policy 39 (3), 1575-1585.

Hobbs, B. F., 1999. LCP Models of Nash-Cournot Competition in Bilateral and POOLCO-Based Power Markets. In: IEEE PES Winter Meeting. Vol. 16. NYC, NY, USA, pp. 194-202.

Hobbs, B. F., Metzler, C. B., Pang, J. S., 2000. Strategic gaming analysis for electric power systems: An MPEC approach. IEEE Transactions on Power Systems 15 (2), 638 - 645.

Huang, Q., Xu, Y., Wang, T., Courcoubetis, C., 2018. Market Mechanisms for Cooperative Operation of Price-maker Energy Storage in a Power Network. IEEE Transactions on Power Systems 33 (3), 3013-3028.

Hutchins, M., 2018. More big batteries on the way in 2018. PV Magazine Australia.

IEC, 2011. Electrical Energy Storage - White Paper. Tech. rep., IEC, Geneva, Switzerland.

IRENA, 2017a. Adapting market design to high shares of variable renewable energy. Tech. rep., IRENA, Abu Dhabi, UAE.

IRENA, 2017b. Electricity storage and renewables: Costs and markets to 2030. Tech. Rep. October, International Renewable Energy Agency, Abu Dhabi, UAE.

Jia, Y., Mi, Z., Yu, Y., Song, Z., Sun, C., 2018. A bilevel model for optimal bidding and offering of flexible load aggregator in day-ahead energy and reserve markets. IEEE Access 6, 67799-67808.

Kardakos, E. G., Simoglou, C. K., Bakirtzis, A. G., 2016. Optimal offering strategy of a virtual power plant: A stochastic bi-level approach. IEEE Transactions on Smart Grid 7 (2), 794-806.

Koirala, B. P., van Oost, E., van der Windt, H., 2018. Community energy storage: A responsible innovation towards a sustainable energy system? Applied Energy 231, 570 - 585. 
Lin, Y., Johnson, J. X., Mathieu, J. L., 2016. Emissions impacts of using energy storage for power system reserves. Applied Energy 168, $444-456$.

McPherson, M., Johnson, N., Strubegger, M., 2018. The role of electricity storage and hydrogen technologies in enabling global low-carbon energy transitions. Applied Energy 216, 649 - 661.

Mohsenian-Rad, H., 2016. Coordinated Price-Maker Operation of Large Energy Storage Units in Nodal Energy Markets. IEEE Transactions on Power Systems 31 (1), 786-797.

Munoz, F. D., Wogrin, S., Oren, S. S., Hobbs, B. F., 2017. Economic Inefficiencies of Cost-Based Electricity Market Designs. USAEE Working Paper No. 17-313.

Nasrolahpour, E., Kazempour, J., Zareipour, H., Rosehart, W. D., 2017. A Bilevel Model for Participation of a Storage System in Energy and Reserve Markets. IEEE Transactions on Sustainable Energy 9 (2), $582-598$.

Nasrolahpour, E., Zareipour, H., Rosehart, W. D., Kazempour, S. J., 2016. Bidding Strategy for an Energy Storage Facility. In: Power Systems Computation Conference (PSCC). Genova, Italy, pp. 1-6.

Nykvist, B., Nilsson, M., 2015. Rapidly falling costs of battery packs for electric vehicles. Nature Climate Change 5 (4), 329-332.

Pandžić, H., Dvorkin, Y., Carrin, M., 2018. Investments in merchant energy storage: Trading-off between energy and reserve markets. Applied Energy 230, $277-286$.

Pandžić, H., Kuzle, I., 2015. Energy storage operation in the day-ahead electricity market. In: International Conference on the European Energy Market (EEM). Lisbon, Portugal, pp. 1-6.

Pandžić, K., Pandžić, H., Kuzle, I., July 2018. Coordination of regulated and merchant energy storage investments. IEEE Transactions on Sustainable Energy 9 (3), 1244-1254.

Pandžić, K., Pandžić, H., Kuzle, I., 2019. Virtual storage plant offering strategy in the day-ahead electricity market. International Journal of Electrical Power \& Energy Systems 104, 401 - 413.

Papavasiliou, A., Mou, Y., Cambier, L., Scieur, D., 2018. Application of Stochastic Dual Dynamic Programming to the Real-Time Dispatch of Storage under Renewable Supply Uncertainty. IEEE Transactions on Sustainable Energy 9 (2), 547-558.

Parvania, M., Fotuhi-Firuzabad, M., Shahidehpour, M., 2014. Comparative hourly scheduling of centralized and distributed storage in day-ahead markets. IEEE Transactions on Sustainable Energy 5 (3), $729-737$.

Peker, M., Kocaman, A. S., Kara, B. Y., 2018. Benefits of transmission switching and energy storage in power systems with high renewable energy penetration. Applied Energy 228, $1182-1197$.

Pérez Arriaga, I., Knittel et al, C., 2016. Utility of the Future. Tech. rep., MIT, Cambridge, MA, USA.

PJM, 2017. PJM Manual 11: Energy and Ancillary Services Market Operations. Tech. rep., PJM, Valley Forge, Pennsylvania, USA.

Ruiz, C., Conejo, A. J., 2009. Pool strategy of a producer with endogenous formation of addressal marginal prices. IEEE Transactions on Power Systems 24 (4), 1855-1866.

Schill, W.-P., Kemfert, C., 2011. Modeling strategic electricity storage: The case of pumped hydro storage in Germany. Energy Journal 32 (3), 59-87.

Schillemans, A., De Vivero-Serrano, G., Bruninx, K., 2018. Strategic participation of merchant energy storage in joint energy-reserve and balancing markets. In: MEDPOWER Proceedings. Dubrovnik, Croatia, pp. $1-6$.

Secretaria de Energia - SENER, 2015. Bases del Mercado Electrico. Tech. rep., SENER, Mexico City, Mexico.

Shahmohammadi, A., Sioshansi, R., Conejo, A. J., Afsharnia, S., 2018a. Market equilibria and interactions between strategic generation, wind, and storage. Applied Energy 220, 876 - 892.

Shahmohammadi, A., Sioshansi, R., Conejo, A. J., Afsharnia, S., 2018b. The role of energy storage in mitigating ramping inefficiencies caused by variable renewable generation. Energy Conversion and Management $162,307-320$.

Sioshansi, R., 2014. When energy storage reduces social welfare. Energy Economics 41, 106 - 116.

Store Project, 2014. Facilitating energy storage to allow high penetration of variable renewable energy Spain Results. Tech. rep., Store Project, Brussels, Belgium.

$\mathrm{Su}, \mathrm{H}$. I., El Gamal, A., 2013. Modeling and analysis of the role of energy storage for renewable integration: Power balancing. IEEE Transactions on Power Systems 28 (4), 4109-4117. 
Taylor, J. A., 2015. Financial storage rights. IEEE Transactions on Power Systems 30 (2), 997-1005.

Taylor, J. A., Callaway, D. S., Poolla, K., 2013. Competitive energy storage in the presence of renewables. IEEE Transactions on Power Systems 28 (2), 985-996.

Vespermann, N., Delikaraoglou, S., Pinson, P., 2017. Offering Strategy of a Price-Maker Energy Storage System in Day-Ahead and Balancing Markets. In: PowerTech IEEE. Manchester, UK, pp. 1-6.

Yang, W., Yang, J., 2019. Advantage of variable-speed pumped storage plants for mitigating wind power variations: Integrated modelling and performance assessment. Applied Energy 237, $720-732$.

Ye, Y., Papadaskalopoulos, D., Moreira, R., Strbac, G., 2017. Strategic capacity withholding by energy storage in electricity markets. In: 2017 IEEE PowerTech. Manchester, UK, pp. 1-6.

Ye, Y., Papadaskalopoulos, D., Strbac, G., 2016. An MPEC approach for analysing the impact of energy storage in imperfect electricity markets. In: International Conference on the European Energy Market, EEM. Lisbon, Portugal, pp. 1-5.

Zou, P., Chen, Q., Xia, Q., He, G., Kang, C., Conejo, A. J., 2016. Pool equilibria including strategic storage. Applied Energy 177, $260-270$.

\section{Appendix A. Bilevel optimization problems}

This appendix contains the bilevel optimization problems that represent the decision problem of a strategic ESS owner participating in electricity markets with different bid structures Appendix A.1 and Appendix A.2 , the corresponding KKT conditions of the lower-level problems required to transform the bilevel problem into an equivalent Mathematical Problem with Equilibrium Constraints (MPEC) Appendix A.3), and a set of mathematical techniques to get rid of the non-linear expressions and reformulate the problem as a mixed integer linear problem (MPEC-MILP) Appendix A.4).

\section{Appendix A.1. Simple bid structures}

Upper level problem:

$$
\max _{x, y, \beta^{q}, \beta^{r}} \sum_{s \in S} \sum_{t \in T} \sum_{k \in K} p_{k} \cdot \pi_{t, k}\left(q_{s, t, k}-r_{s, t, k}\right)
$$

subject to

$$
\begin{aligned}
& \underline{v}_{s} \leq v_{s, t, k} \leq \bar{v}_{s} \quad \forall s \in S, \forall t \in T, \forall k \in K \\
& v_{s, t, k}=v_{s, t-1, k}+\delta_{s}^{c} r_{s, t, k}-1 / \delta_{s}^{d} q_{s, t, k} \quad \forall s \in S, \forall t \in T, \forall k \in K \\
& v_{s, t=0, k}=v_{s, t=T, k} \quad \forall s \in S, \forall k \in K \\
& 0 \leq x_{s, t} \leq \bar{q}_{s} \quad \forall s \in S, \forall t \in T \\
& 0 \leq y_{s, t} \leq \bar{r}_{s} \quad \forall s \in S, \forall t \in T \\
& 0 \leq \beta_{s, t}^{q} \leq \Pi \quad \forall s \in S, \forall t \in T \\
& 0 \leq \beta_{s, t}^{r} \leq \Pi \quad \forall s \in S, \forall t \in T \\
& q_{s, t, k}, r_{s, t, k}, \pi_{t, k}=\operatorname{argmin} \mathcal{L}_{k}\left(x_{s, t}, y_{s, t}, \beta_{s, t}^{q}, \beta_{s, t}^{r}\right) \quad \forall k \in K
\end{aligned}
$$


Lower level problem: The model below represents the problem for each scenario $k$ (set $K$ ):

$$
\min \mathcal{L}_{k}\left(x_{s, t}, y_{s, t}, \beta_{s, t}^{q}, \beta_{s, t}^{r}\right)=\min \sum_{t \in T}\left(\sum_{s \in S} \beta_{s, t}^{q} \cdot q_{s, t, k}-\sum_{s \in S} \beta_{s, t}^{r} \cdot r_{s, t, k}+\sum_{i \in I} c_{i, t} \cdot g_{i, t, k}+c^{\phi} \cdot \phi_{t, k}\right)
$$

subject to

$$
\begin{aligned}
& \sum_{i \in I} g_{i, t, k}+\sum_{j \in J} w_{j, t, k}+\sum_{s \in S} q_{s, t, k}-\sum_{s \in S} r_{s, t, k}+\phi_{t, k}=D_{t} \quad:\left[\pi_{t, k}\right] ; \forall t \in T \\
& 0 \leq q_{s, t, k} \leq x_{s, t} \quad:\left[\underline{\mu}_{t, k}, \bar{\mu}_{t, k}\right] ; \quad \forall s \in S, \forall t \in T \\
& 0 \leq r_{s, t, k} \leq y_{s, t} \quad:\left[\underline{\lambda}_{t, k}, \bar{\lambda}_{t, k}\right] ; \quad \forall s \in S, \forall t \in T \\
& 0 \leq w_{j, t, k} \leq \bar{w}_{j, t, k} \quad:\left[\underline{\psi}_{t, k}, \bar{\psi}_{t, k}\right] ; \quad \forall j \in J, \forall t \in T \\
& 0 \leq g_{i, t, k} \leq \bar{g}_{i} \quad:\left[\underline{\theta}_{t, k}, \bar{\theta}_{t, k}\right] ; \quad \forall i \in I, \forall t \in T \\
& 0 \leq \phi_{t, k} \leq D_{t} \quad:\left[\underline{\phi}_{t, k}, \bar{\phi}_{t, k}\right] ; \quad \forall t \in T
\end{aligned}
$$

Appendix A.2. Complex bid structure

Upper level problem.

$$
\max _{x, y} \sum_{s \in S} \sum_{t \in T} \sum_{k \in K} p_{k} \cdot \pi_{t, k}\left(q_{s, t, k}-r_{s, t, k}\right)
$$

subject to

$$
\begin{aligned}
& 0 \leq x_{s, t} \leq \bar{q}_{s} \quad \forall s \in S, \forall t \in T \\
& 0 \leq y_{s, t} \leq \bar{r}_{s} \quad \forall s \in S, \forall t \in T \\
& q_{s, t, k}, r_{s, t, k}, \pi_{t, k}=\operatorname{argmin} \mathcal{L}_{k}\left(x_{s, t}, y_{s, t}\right) \quad \forall k \in K
\end{aligned}
$$

Lower level problem. The model below represents the problem for each scenario $k$ (set $K)$ :

$$
\min \mathcal{L}_{k}\left(x_{s, t}, y_{s, t}\right)=\min \sum_{t \in T}\left(\sum_{i \in I} c_{i, t} \cdot g_{i, t, k}+c^{\phi} \cdot \phi_{t, k}\right)
$$


subject to

$$
\begin{aligned}
& \sum_{i \in I} g_{i, t, k}+\sum_{j \in J} w_{j, t, k}+\sum_{s \in S} q_{s, t, k}-\sum_{s \in S} r_{s, t, k}+\phi_{t, k}=D_{t} \quad:\left[\pi_{t, k}\right] ; \quad \forall t \in T \\
& 0 \leq q_{s, t, k} \leq x_{s, t} \quad:\left[\underline{\mu}_{t, k}, \bar{\mu}_{t, k}\right] ; \quad \forall s \in S, \forall t \in T \\
& 0 \leq r_{s, t, k} \leq y_{s, t} \quad:\left[\underline{\lambda}_{t, k}, \bar{\lambda}_{t, k}\right] ; \quad \forall s \in S, \forall t \in T \\
& 0 \leq g_{i, t, k} \leq \bar{g}_{i} \quad:\left[\underline{\theta}_{t, k}, \bar{\theta}_{t, k}\right] ; \quad \forall i \in I, \forall t \in T \\
& 0 \leq w_{j, t, k} \leq \bar{w}_{j, t, k} \quad:\left[\underline{\psi}_{t, k}, \bar{\psi}_{t, k}\right] ; \quad \forall j \in J, \forall t \in T \\
& 0 \leq \phi_{t, k} \leq D_{t} \quad:\left[\underline{\phi}_{t, k}, \bar{\phi}_{t, k}\right] ; \quad \forall t \in T \\
& \underline{v}_{s} \leq v_{s, t, k} \leq \bar{v}_{s} \quad:\left[\underline{\sigma}_{t, k}, \bar{\sigma}_{t, k}\right] ; \quad \forall s \in S, \forall t \in T \\
& v_{s, t, k}=v_{s, t-1, k}+\delta_{s}^{c} r_{s, t, k}-1 / \delta_{s}^{d} q_{s, t, k} \quad:\left[\alpha_{s, t, k}\right] ; \quad \forall s \in S, \forall t \in T \\
& v_{s, t=0, k}=v_{s, t=T, k} \quad:\left[\sigma_{s, k}^{T}\right] ; \quad \forall s \in S
\end{aligned}
$$

Appendix A.3. Karush-Kuhn Tucker (KKT) conditions of the lower-level problem

The lower level problem A.10- A.16), representing the market clearing problem under the simple bid structures, can be replaced by the associated KKT conditions:

Stationary conditions.

$$
\begin{array}{ll}
c_{i, t}-\pi_{t, k}-\underline{\theta}_{i, t, k}+\bar{\theta}_{i, t, k}=0 & \forall i \in I, \forall t \in T, \forall k \in K \\
c_{j}^{w}-\pi_{t, k}-\underline{\psi}_{j, t, k}+\bar{\psi}_{j, t, k}=0 & \forall j \in J, \forall t \in T, \forall k \in K \\
\beta_{s, t}^{q}-\pi_{t, k}-\underline{\mu}_{s, t, k}+\bar{\mu}_{s, t, k}=0 & \forall s \in S, \forall t \in T, \forall k \in k \\
-\beta_{s, t}^{r}+\pi_{t, k}-\underline{\lambda}_{s, t, k}+\bar{\lambda}_{s, t, k}=0 & \forall s \in S, \forall t \in T, \forall k \in K \\
c^{\phi}-\pi_{t, k}-\underline{\phi}_{t, k}+\bar{\phi}_{t, k}=0 & \forall t \in T, k \in K
\end{array}
$$

Complementary slackness conditions.

$$
\begin{aligned}
& 0 \leq g_{i, t, k} \perp \underline{\theta}_{i, t, k} \geq 0 \quad \forall i \in I, \forall t \in T, k \in K \\
& 0 \leq w_{j, t, k} \perp \underline{\psi}_{j, t, k} \geq 0 \quad \forall j \in J, \forall t \in T, k \in K \\
& 0 \leq q_{s, t, k} \perp \underline{\mu}_{s, t, k} \geq 0 \quad \forall s \in S, \forall t \in T, \forall k \in K \\
& 0 \leq r_{s, t, k} \perp \underline{\lambda}_{s, t, k} \geq 0 \quad \forall s \in S, \forall t \in T, \forall k \in K \\
& 0 \leq \phi_{t, k} \perp \underline{\phi}_{t, k} \geq 0 \quad \forall t \in T, k \in K \\
& 0 \leq \bar{g}_{i}-g_{i, t, k} \perp \bar{\theta}_{i, t, k} \geq 0 \quad \forall i \in I, \forall t \in T, k \in K \\
& 0 \leq \bar{w}_{j, t, k}-w_{j, t, k} \perp \bar{\psi}_{j, t, k} \geq 0 \quad \forall j \in J, \forall t \in T, k \in K \\
& 0 \leq x_{s, t}-q_{s, t, k} \perp \bar{\mu}_{s, t, k} \geq 0 \quad \forall s \in S, \forall t \in T, \forall k \in K \\
& 0 \leq y_{s, t}-r_{s, t, k} \perp \bar{\lambda}_{s, t, k} \geq 0 \quad \forall s \in S, \forall t \in T, \forall k \in K \\
& 0 \leq D_{t}-\phi_{t, k} \perp \bar{\phi}_{t, k} \geq 0 \quad \forall t \in T, k \in K
\end{aligned}
$$


The KKT conditions associated with the lower level problem of the bilevel problem representing the complex bid structure (A.21)-A.30) are identical to Equations (A.31)A.45, with following exceptions and additions:

Stationary conditions. Equations (A.38)-A.38) are substituted by Equations (A.46)-(A.47):

$$
\begin{array}{cc}
-\pi_{t, k}-\underline{\mu}_{s, t, k}+\bar{\mu}_{s, t, k}-1 / \delta_{s}^{d} \cdot \alpha_{s, t, k}=0 & \forall s \in S, \forall t \in T, \forall k \in K \\
\pi_{t, k}-\underline{\lambda}_{s, t, k}+\bar{\lambda}_{s, t, k}+\delta_{s}^{c} \cdot \alpha_{s, t, k}=0 & \forall s \in S, \forall t \in T, \forall k \in K
\end{array}
$$

Moreover, the following stationary conditions are added:

$$
\begin{aligned}
& \alpha_{s, t+1, k}+\sigma_{s, k}^{T}-\underline{\sigma}_{s, t, k}+\bar{\sigma}_{s, t, k}=0 \quad \forall s \in S, \forall k \in K, t=0 \\
& -\alpha_{s, t, k}+\alpha_{s, t+1, k}-\underline{\sigma}_{s, t, k}+\bar{\sigma}_{s, t, k}=0 \quad \forall s \in S, \forall k \in K, \forall t=1: T-1 \\
& -\alpha_{s, t, k}-\sigma_{s, k}^{T}-\underline{\sigma}_{s, t, k}+\bar{\sigma}_{s, t, k}=0 \quad \forall s \in S, \forall k \in K, t=T
\end{aligned}
$$

Complementary slackness conditions. In addition to Equations (A.36)-(A.45), the following complementary slackness conditions need to be considered under the complex bid structure:

$$
\begin{array}{ll}
0 \leq v_{s, t, l}-\underline{v}_{s} \perp \underline{\sigma}_{s, t} \geq 0 & \forall s \in S, \forall t \in T, \forall l \in L \\
0 \leq \bar{v}_{s}-v_{s, t, l} \perp \bar{\sigma}_{s, t} \geq 0 & \forall s \in S, \forall t \in T, \forall l \in L
\end{array}
$$

Appendix A.4. Linear equivalents of non-linear expressions

By replacing the lower level problem by the associated KKT conditions, the bilevel programming model is recast as an equivalent Mathematical Problem with Equilibrium Constraints (MPEC). The resulting MPEC formulation contains several non-linear expressions in the objective function and in the complementary slackness conditions, which are linearized as shown in Appendix A.4.1 and Appendix A.4.2. The resulting problems are Mixed Integer Linear Programming problems, which can be solved with off-the-shelf solvers, such as CPLEX or Gurobi.

Appendix A.4.1. Non-linear expressions in the objective function of the upper-level problem

The objective function of the MPEC formulations (A.1) and A.17) is a non-linear expression due to the product of charging and discharging variables with the market price. This expression is linearized by means of the strong duality theorem applied to the lower level problem and a set of KKT conditions (Equations (A.33), (A.34), A.38), (A.39), (A.43) and (A.44) $)$. The resulting linear objective function, replacing Equations (A.1) and (A.17), reads:

$$
\begin{aligned}
& \max _{x, y, \beta^{q}, \beta^{r}} \sum_{k \in K} p_{k} \cdot \sum_{t \in T}\left(D_{t}\left(\pi_{t, k}-\bar{\phi}_{t, k}\right)-c^{\phi} \cdot \phi_{t, k}\right) \\
& -\sum_{k \in K} p_{k} \cdot \sum_{i \in I} \sum_{t \in T}\left(c_{i, t} \cdot g_{i, t, k}+\bar{g}_{i} \cdot \bar{\theta}_{i, t, k}\right)-\sum_{k \in K} p_{k} \cdot \sum_{j \in J} \sum_{t \in T}\left(\bar{w}_{j, t, k} \cdot \bar{\psi}_{j, t, k}\right)
\end{aligned}
$$


Appendix A.4.2. Non-linear expressions in the complementary slackness conditions of the lower-level problem

The non-linear expressions present in the complementary slackness conditions are recast as a set of mixed-integer linear programming constraints using the Big-M method (FortunyAmat and McCarl, 1981). For the sake of conciseness, we present only a set of constraints corresponding to the complementary conditions associated to the lower and upper bounds of the discharging power (Equation A.23 with complementary slackness conditions A.38 and $(\mathrm{A.43})$ ). The procedure is identical for the other complementary slackness conditions.

$$
\begin{aligned}
q_{s, t, k}, \underline{\mu}_{s, t, k} \geq 0 & \forall s \in S, \forall t \in T, \forall k \in K \\
q_{s, t, k} \leq M \cdot B \underline{\mu}_{s, t, k} & \forall s \in S, \forall t \in T, \forall k \in K \\
\underline{\mu}_{s, t, k} \leq M\left(1-B \underline{\mu}_{s, t, k}\right) & \forall s \in S, \forall t \in T, \forall k \in K \\
B \underline{\mu}_{s, t, k} \in\{0,1\} & \forall s \in S, \forall t \in T, \forall k \in K \\
x_{s, t}-q_{s, t, k} \geq 0 \quad \bar{\mu}_{s, t, k} \geq 0 & \forall s \in S, \forall t \in T, \forall k \in K \\
x_{s, t}-q_{s, t, k} \leq M \cdot B \bar{\mu}_{s, t, k} & \forall s \in S, \forall t \in T, \forall k \in K \\
\bar{\mu}_{s, t, k} \leq M\left(1-B \bar{\mu}_{s, t, k}\right) & \forall s \in S, \forall t \in T, \forall k \in K \\
B \bar{\mu}_{s, t, k} \in\{0,1\} & \forall s \in S, \forall t \in T, \forall k \in K
\end{aligned}
$$

